\title{
Efficacy of personalized models in discriminating high cognitive demand conditions using text-based interactions
}

\author{
Lisa M. Vizer ${ }^{\mathrm{a}, 1}$ (Corresponding Author) \\ Andrew Sears ${ }^{\text {a,2 }}$ \\ ${ }^{a}$ UMBC, 100 Hilltop Cir., Baltimore, MD 21250 USA \\ lisa_vizer@unc.edu \\ andrew.sears@psu.edu
}

\begin{abstract}
Although high cognitive demand conditions can impair psychological, physical, and behavioral processes without appropriate management, current measurement methods are too cumbersome for continuous monitoring of cognitive demand, and do not account for individual differences. This research uses keystroke and linguistic markers of typed text to construct individualized models of cognitive demand response to discriminate high and low cognitive demand conditions, the results of which can have implications for design of cognitive demand monitoring systems for personalized health management. We constructed within-subject models of cognitive demand response for nine participants and one between-subjects model based on 20 participants. The AUCs for personalized models ranged from 0.679 to 0.953 (Mean=0.826, $\mathrm{SD}=0.085$ ), significantly higher than chance $(\mathrm{p}<0.0001)$ and the 0.714 AUC for the generic model $(\mathrm{p}=0.002)$. Although the features in each model were different, the most common features across models are rate of negative emotion, lexical diversity, rate of words over six letters, and word count. These results confirm significant individual differences in cognitive demand response and suggest that measurement methods used in a monitoring system must adapt to individual characteristics. Our research operationalizes the effects of cognitive demand on HCI and contributes a unique combination of text and keystroke features used to detect high cognitive demand situations.
\end{abstract}

Key Words: Cognitive load, Cognitive stress, Cognitive demand, Consumer health informatics, Health monitoring, Human-centered computing

\section{INTRODUCTION}

High cognitive demand is a part of everyday life for individuals working in stressful and challenging roles, ranging from high-pressure office work to critical airtraffic control. Consequences for people can include impaired judgment and memory, sleep and motor disturbances, and difficulty performing self-care tasks (Lupien et al. 2007; Semmer et al. 2005). Furthermore, stress produces a high public health burden (American Psychological Association 2015), accounting for up to $\$ 190$ billion in health care expenditures alone (Goh et al. 2015). This figure does not capture the additional burdens of absenteeism, lowered productivity, and unemployment that add to the public health burden. However, people can mitigate the harmful psychological, physical, behavioral, and performance consequences of high cognitive demand if they engage in healthy stress management behaviors such as relaxation exercises (Varvogli and Darviri 2011).

\footnotetext{
${ }^{1}$ L.M. Vizer, (Current address) University of North Carolina at Chapel Hill, Department of Medicine, Campus Box 7110, Chapel Hill, NC, 27599 USA

${ }^{2}$ A. Sears, (Current address) Pennsylvania State University, College of Information Sciences and Technology, 201 Old Main, University Park, PA 16802 USA
} 
A barrier to enacting such timely interventions is a lack of adequate methods for detecting high demand in real time to prompt interventions. The most commonly used indicators in tests administered to assess cognitive demand include physiological signs, self-report responses, behavioral measures, and performance measures. These tools are valid and reliable for discrete testing that identifies functioning outside of normative standards but have drawbacks when it comes to personalized real-time measurement and change detection. In particular, existing assessments are often obtrusive, resource intensive, impractical for real-time identification of acute cognitive demand, and do not account for individual differences.

Furthermore, these tests are not integrated into pervasive and familiar contexts, such as every day information technology (IT) interaction. There is a huge opportunity for evolving cognitive demand assessment tools to take advantage of the ubiquity of IT interactions in everyday life. According to the Pew Research Internet Project, as of November 2012 78\% of Americans own either a laptop or desktop computer (Pew Research Internet Project 2014). The pervasiveness of technology makes monitoring of computer interactions a compelling platform for detecting conditions of high cognitive demand, and affords access to a wider audience than can smartphones at $64 \%$ ownership (Smith 2015), for example. The recent trend in using technology for self-monitoring (Fox and Duggan 2013) also offers a complement to conventional measures.

This research is based upon the premise that an individual's typing patterns are generally stable, but can vary in demanding situations (Monrose and Rubin 2000). Prior research in human-computer interaction supports the link between changes in cognitive demand and changes in IT interactions (Vizer et al. 2009; Vizer 2013; Vizer and Sears, 2015). Vizer et al. $(2009,2013)$ found that cognitive and physical stress affect linguistic and keystroke attributes of typed text. Further research also observed changes in attributes of typed text related to cognitive impairment in older adults (Vizer and Sears, 2015). Despite the ubiquity of information technology and the large public health burden related to high cognitive demand (American Psychological Association 2015; Goh et al. 2015), we have yet to fully explore how to develop personalized models to passively detect high levels of cognitive demand in order to implement appropriate support and interventions. New monitoring techniques using commonplace computer interactions could allow introduction of effective interventions for reducing or even preventing the acute and chronic impacts of high cognitive demand (Lupien et al. 2007; Semmer et al. 2005). Once a system detects the presence of high cognitive demand, it could introduce appropriate interventions. For those in critical high demand situations the system could introduce interface adaptations or task modulation to maintain precision and accuracy despite the high-pressure environment. For everyday high demand, such as an office worker might encounter, the system could prompt a stress-reduction activity such as deep breathing.

Information technology offers a unique vehicle for monitoring since computer and technology use is widespread and growing. The cognitive processes needed to use technology and those impacted by cognitive demand are related (Card et al. 1986), including memory, perception, attention, and language. Furthermore, current assessments prove too obtrusive and resource intensive when applied to the problem of detecting acute high cognitive demand conditions. The goal of this study is to understand how acute high cognitive demand affects text-based interactions in real time with everyday information technology. This is a first step in developing approaches to passively monitor cognitive function using everyday computer interactions. In this research we leverage the ubiquity of keyboard interactions to 
study how changes in keystroke and linguistic attributes of typed text are related to acute cognitive demand.

Earlier research has employed either keystroke features or linguistic features for high cognitive demand detection, but this study examines the combination for classifying cognitive demand status in individuals. This novel combination gives us a more nuanced understanding of how high cognitive demand is expressed in interactions with a computer than only keystroke or linguistic features alone. Results show that personalized models are more powerful than a generic model in detecting the unique ways individuals react to high cognitive demand.

\section{RELATED LITERATURE}

Insights from literature on cognitive function, cognitive assessment, and methods of text analysis illustrate the possibility of leveraging everyday IT use for unobtrusive monitoring of keyboard interactions for evidence of high cognitive demand. First is a short overview of how cognitive demands impact cognitive function. Next, current methods of assessing cognitive function are reviewed. Finally, we survey language analysis research to identify features of typed text that can fluctuate in response to increased demand. Opportunities for applying these domains to the goal of real-time, unobtrusive monitoring motivate this research.

\subsection{Cognitive demand}

Cognitive demand describes objective characteristics of a task or situation that demands effort on the part of a person (Mehler et al. 2012). This demand results in that person experiencing some degree of cognitive stress and cognitive load (Hone and Graham 2000), which both affect cognitive functioning. For this study, we define high cognitive demand as an amount of cognitive stress and cognitive load that an individual experiences as taxing or overwhelming.

Cognitive stress is an individual's physiological and psychological response to a stressor that she perceives as threatening or taxing and overwhelming to her ability to cope (Goldberger and Breznitz 2010). A moderate amount of stress can improve performance, but too much will degrade performance (Lupien et al. 2007),

Cognitive load is the perception of the total cognitive effort expended on a task (Sweller 1988). As described with cognitive stress, increasing amounts of load will improve performance to a point at which it begins to degrade (Sweller 1988). Sweller introduced Cognitive Load Theory (CLT) as a means of improving instructional design and, in turn, learning. However, this theory is applicable to other situations in which people function under high cognitive load. Oviatt (2006) uses this theory to design human-centered user interfaces that are more intuitive and reduce user error by "freeing up" cognitive resources.

The amount of cognitive demand induced by a given situation will vary from person to person because when different people are in a similar situation each reacts differently. For example, some individuals may find a given situation threatening while others find it only a nuisance. The interaction of each individual's unique characteristics with features of his or her environment and health affect performance on tasks when under demand. For example, Eysenck et al. (2007) review research on the impact of trait anxiety on task performance, finding significant differences in task performance between persons with high trait anxiety versus those with low trait anxiety. Findings suggest that individual differences in trait anxiety affect components of working memory and attentional control in demanding situations, thus impacting performance. 
A high cognitive demand task can cause changes in cognitive function, including deficits in concentration, memory, reasoning, and verbal functioning (Lupien et al. 2007). Each of these cognitive functions is utilized during interactions with IT, as illustrated by Card, Moran, and Newell's Model Human Processor (Card et al. 1986). Since cognitive functions are utilized extensively during interactions with IT, we expect that cognitive demand factors will also impact performance on tasks involving information technologies and that those performance impacts will vary from person to person. These observations motivate this study's investigation of the effects of cognitive demand on text-based technology interactions.

\subsection{Assessment of cognitive demand conditions}

The effects of cognitive demand are chiefly measured through physiological signs, self-assessments, and performance measures (Bourne and Yaroush 2003; Wilson 2002). However, each of these tests suffers from one or more of the following drawbacks: requiring specialized training, requiring specialized equipment, obtrusiveness, timeintensiveness, inconvenience, and impracticality for frequent administration (Bourne and Yaroush 2003).

Physiological measurements are the most common method for detecting changes in cognitive demand. Increases in stress hormone levels, heart rate, blood pressure (Cohen et al. 1995), pupil dilation, and galvanic skin response (Goldberger and Breznitz 2010) can all indicate the presence of increased stress and load. However, proper equipment, training, and interpretation are necessary to ensure accurate results. The assessments can be obtrusive, require supplementary equipment, and are often labor or computationally intensive. One example is measurement of driver stress using a car instrumented with various physiological sensors (Healey and Picard 2000). The car's sensors achieve high correct classification rates for high driver stress, but the sensors and computing equipment are impractical for widespread use. Our research aims to use widely available technology for monitoring.

Self-report instruments for demand are subjective questionnaires that assess the level of workload, distress, and other indicators. Examples include the NASA-TLX (Hart and Staveland 1988), the Pressure Management Inventory (Williams and Cooper 1998), the Dundee Stress State Questionnaire (Matthews et al. 2002), and the Job Content Questionnaire (Karasek et al. 1998). The NASA-TLX, in particular, is extensively researched and validated, takes very little time to administer, and is robust under diverse administration circumstances. These questionnaires are sensitive to the experience of demand, but require interruption of the person's primary task. We are interested in developing a method that requires no extra effort on the part of the user beyond the activities in which they are already engaged.

Performance measures are a means of gauging function under stress and load through overt output on a primary or secondary task. Common methods for manipulating load and stress on a given task include varying type and amount of load, varying speed, or introducing a secondary task (Gawron 2000). Measures of accuracy, errors, and efficiency capture the level of execution in a situation of high stress or load. The effects of load and stress on performance are extensively studied (Goldberger and Breznitz 2010; Mandler 2010), but results are inconclusive, with researchers observing increases, decreases, and no differences in performance. This research uses keystroke and linguistic features as performance measures to gauge cognitive demand.

HCI researchers have explored various means of using behavior on interaction tasks as performance measures for detecting high cognitive demand conditions. Carneiro et al. (2012) used performance features to detect the presence of several levels of experimentally induced cognitive stress in interaction samples using a 
mouse. The most predictive features were acceleration and touch pressure. This study also showed significant individual differences in the stress response. They conclude that although a general model is useful when training data is not available, a personalized model is more accurate. This is consistent with Hancock's model of adaptability under stress (Hancock 1989). We build on the work of Carneiro et al. by exploring individual differences in the load and stress response as measured by keystroke and linguistic features.

In two studies Mark et al. $(2008,2012)$ find that faster work pace is accompanied by higher experience of stress. In the first study (Mark et al. 2008), workers completed interrupted tasks faster, but experienced increased stress, frustration, and effort. The second study (Mark et al. 2012) found that by eliminating email participants reported less stress and better task focus. Although cutting off email indefinitely may not be feasible, the results of these studies highlight benefits of better workflow management with regard to optimizing task completion and stress levels.

$\mathrm{Lu}$ et al. (2012) investigate the use of speech to detect stress features in real-time on a cell phone. This method is unobtrusive as it can monitor normal conversations, but is computationally intensive. They achieve up to $81 \%$ accuracy using optimum feature sets and acoustic conditions.

Adams et al. (2014) explored minimally intrusive methods for monitoring stress, including physiological sensors, voice metrics, and self-report measures. They found that all methods detect stress and were relatively unobtrusive, but participants had concerns about each. Sensors could be uncomfortable or noticed by others, and were expensive. Voice analysis was computationally expensive and participants were concerned with privacy despite the absence of recording. Self-report prompts required some effort for response and could be disruptive.

The research presented in this paper tests a method for detecting cognitive demand that addresses some of the shortcomings of other methods. It is unobtrusive, requires no special equipment, requires little computing power, requires no extra effort on the part of the person assessed, and is practical for continuous monitoring.

\subsection{Language and Keystroke Features Under High Cognitive Demand}

The preceding section demonstrated opportunities for improvement over traditional cognitive assessments. One approach is to monitor function using linguistic and keystroke analyses of a person's routine text-based computer interactions as a type of performance measure. We look at two bodies of literature. First, we examine literature on language changes under high cognitive load, and then we survey literature on language changes under one type of high cognitive stress. At least one prior study has shown that analysis of keyboard interactions can also identify evidence of high cognitive stress using both keystroke and language features.

\subsubsection{Language and cognitive load}

To ascertain differences indicative of high cognitive load, researchers analyze language produced while a person is under high cognitive load and compare it to language produced under low cognitive load by examining transcripts of spoken interactions. Predictions concerning features distinguishing language produced under high versus low cognitive load are largely based on Baddeley's theory of working memory and language (Baddeley 2003). This theory explains many of the language changes under high cognitive load as the result of a decrease in available working memory. Table I summarizes the timing and language features predicted to 
change under high cognitive load, as informed by the literature on language under cognitive load.

Table I. Timing and language features predicted to change under high cognitive load conditions

\begin{tabular}{|c|c|c|}
\hline CATEGORY & FEATURE & DEFINITION \\
\hline \multirow[t]{3}{*}{ Timing } & Duration $^{3}$ & total elapsed time for task \\
\hline & Pause rate ${ }^{3}$ & total \# pauses / total \# words \\
\hline & Mean pause duration ${ }^{3}$ & total pause time / total \# pauses \\
\hline \multirow[t]{3}{*}{ Language complexity } & Word count ${ }^{1}$ & total \# of words \\
\hline & Mean sentence length ${ }^{1}$ & total \# words / total \# sentences \\
\hline & Words over six letters ${ }^{1}$ & $\begin{array}{l}\text { total \# words with six or more letters / } \\
\text { total \# words }\end{array}$ \\
\hline Lexical diversity & Unique word rate ${ }^{1}$ & total \# unique words / total \# words \\
\hline \multirow[t]{2}{*}{ Cognitive complexity } & Cognitive processes rate ${ }^{4}$ & total \# cognition words / total \# words \\
\hline & & $\begin{array}{l}\text { Cognition words express cognitive operations } \\
\text { such as thoughts and reasoning. }\end{array}$ \\
\hline \multirow[t]{2}{*}{ Sensing } & Perception word rate ${ }^{4}$ & total \# sensory words / total \# words \\
\hline & & $\begin{array}{l}\text { Sensory words express sensorial experiences } \\
\text { such as seeing, hearing, and feeling. }\end{array}$ \\
\hline \multirow[t]{4}{*}{ Affect } & Positive emotion rate ${ }^{2}$ & total \# positive affect words / total \# words \\
\hline & & $\begin{array}{l}\text { Positive affect is defined as conscious } \\
\text { subjective aspect of a positive emotion apart } \\
\text { from bodily changes. }\end{array}$ \\
\hline & Negative emotion rate ${ }^{2}$ & total \# negative affect words / total \# words \\
\hline & & $\begin{array}{l}\text { Negative affect is defined as conscious } \\
\text { subjective aspect of a negative emotion apart } \\
\text { from bodily changes. }\end{array}$ \\
\hline \multirow[t]{4}{*}{ Personal pronouns } & First-person singular pronouns ${ }^{2}$ & $\begin{array}{l}\text { total \# first-person singular pronouns / } \\
\text { total \# words }\end{array}$ \\
\hline & Third-person singular pronouns ${ }^{2}$ & $\begin{array}{l}\text { total \# third-person singular pronouns / } \\
\text { total \# words }\end{array}$ \\
\hline & First-person plural pronouns ${ }^{2}$ & $\begin{array}{l}\text { total \# first-person plural pronouns / } \\
\text { total \# words }\end{array}$ \\
\hline & Third-person plural pronouns ${ }^{2}$ & $\begin{array}{l}\text { total \# third-person plural pronouns / } \\
\text { total \# words }\end{array}$ \\
\hline \multicolumn{3}{|c|}{$\begin{array}{l}{ }^{2} \text { Khawaja et al. } 2013 \\
{ }^{3} \text { Schilperoord } 2002 \\
{ }^{4} \text { Sweller et al. } 2011\end{array}$} \\
\hline
\end{tabular}

When a speaker is under high cognitive load, studies find that he takes more time overall and pauses more often and for a longer amount of time (Schilperoord 2002). Language complexity increases as short-term memory is overloaded (Khawaja and Chen 2010). Conversely, language diversity decreases for the same reason (Khawaja and Chen 2010). The use of words indicating cognition, such as "think" or 
"know", and sensing increase as people are engaged in increased mental effort (Sweller et al. 2011). Incidence of words indicating positive emotion decreases and incidence of words indicating negative emotion increases since high cognitive load can induce frustration (Khawaja et al. 2013). Under high cognitive load, and especially during collaboration, the use of singular pronouns decreases while the use of plural pronouns increases (Khawaja et al. 2013).

Although research in this area focuses on transcripts of speech, the results may be generalizable to text input using a computer. Our research leverages insights from analysis of transcripts of speech to inform analysis of typed text.

\subsubsection{Language and cognitive stress}

One aspect of deception detection research is the study of how language changes during deceptive tasks, which are highly stressful (Zhou et al. 2003). As with analysis of language produced under high cognitive load, transcripts and recordings are analyzed for evidence of high cognitive stress. Table II summarizes the timing and language features predicted to change under high cognitive stress, as informed by the literature on deception detection as a proxy for language under cognitive stress.

Table II. Timing and language features predicted to change under high cognitive stress deception conditions

\begin{tabular}{|c|c|c|}
\hline CATEGORY & FEATURE & DEFINITION \\
\hline Timing & $\begin{array}{l}\text { Duration }^{2} \\
\text { Pause rate } \\
\text { Mean pause duration }\end{array}$ & $\begin{array}{l}\text { total elapsed time for task } \\
\text { total \# pauses / total \# words } \\
\text { total pause time / total \# pauses }\end{array}$ \\
\hline Informality & Self-correction rate ${ }^{3}$ & total \# self-corrections / total \# words \\
\hline Language complexity & $\begin{array}{l}\text { Word count }{ }^{2} \\
\text { Mean sentence length } \\
\\
\text { Words over six letters }\end{array}$ & $\begin{array}{l}\text { total \# of words } \\
\text { total \# words / total \# sentences } \\
\text { total \# words with six or more letters / } \\
\text { total \# words }\end{array}$ \\
\hline Lexical diversity & Unique word rate ${ }^{2}$ & total \# unique words / total \# words \\
\hline Cognitive complexity & $\begin{array}{l}\text { Cognitive processes rate }{ }^{3} \\
\text { Certainty rate }{ }^{2}\end{array}$ & $\begin{array}{l}\text { total \# cognition words / total \# words } \\
\text { total \# certainty words / total \# words } \\
\text { Certainty words express conviction. }\end{array}$ \\
\hline Affect & $\begin{array}{l}\text { Positive emotion rate }{ }^{2} \\
\text { Negative emotion rate }\end{array}$ & $\begin{array}{l}\text { total \# positive affect words / total \# words } \\
\text { total \# negative affect words / total \# words }\end{array}$ \\
\hline Personal pronouns & $\begin{array}{l}\text { First-person singular } \\
\text { pronouns }^{2}\end{array}$ & $\begin{array}{l}\text { total \# first-person singular pronouns / } \\
\text { total \# words }\end{array}$ \\
\hline $\begin{array}{l}{ }^{1} \text { DePaulo et al. } 2003 \\
{ }^{2} \text { Zhou et al. } 2003 \\
{ }^{3} \text { Zhou } 2005\end{array}$ & & \\
\hline
\end{tabular}

Many changes are consistent with those predicted by the literature on language under cognitive load, but some are not (Zhou et al. 2003; Zuckerman and Driver 1985; DePaulo et al. 2003). Deception detection research tells us that utterances will employ shorter sentences, shorter words, and more positive affect words under cognitive stress, unlike the predictions from research on language under cognitive 
load (Zhou and Zhang 2008). In addition, studies show a decrease in certainty (Zhou and Zhang 2008), a subcategory of cognitive complexity not explored in the cognitive load literature.

Although most of the work in deception detection is based on transcripts of speech, some work using computer-mediated communication shows that results are generalizable to computer interactions (Zhou and Zhang 2006; Zhou et al. 2004). Our work compares results to determine efficacy of the features in Table II as indicators of cognitive stress during IT interactions.

\subsubsection{Information technology interactions and cognitive demand}

The reader is referred to Kolakowska (2013) for an overview of the methods and results of several studies using keystroke dynamics and mouse movements for detecting a variety of emotions. Here we present some of the most relevant research.

In a study classifying text samples produced under high cognitive demand, Vizer et al. (2009) confirmed a link between the presence of cognitive demand and changes in keystroke and linguistic features of spontaneously typed text. Using timing features, keystroke rates, and features of language, they constructed models of cognitive demand for a group of participants. They obtained $75 \%$ classification accuracy, an improvement over a chance rate of $50 \%$, for high demand versus low demand samples using machine learning in a within subjects study. Keystroke and linguistic features used in the machine learning models are listed in Table III.

Table III. Timing and language features predicted to change under high cognitive demand conditions from Vizer et al. (2009)

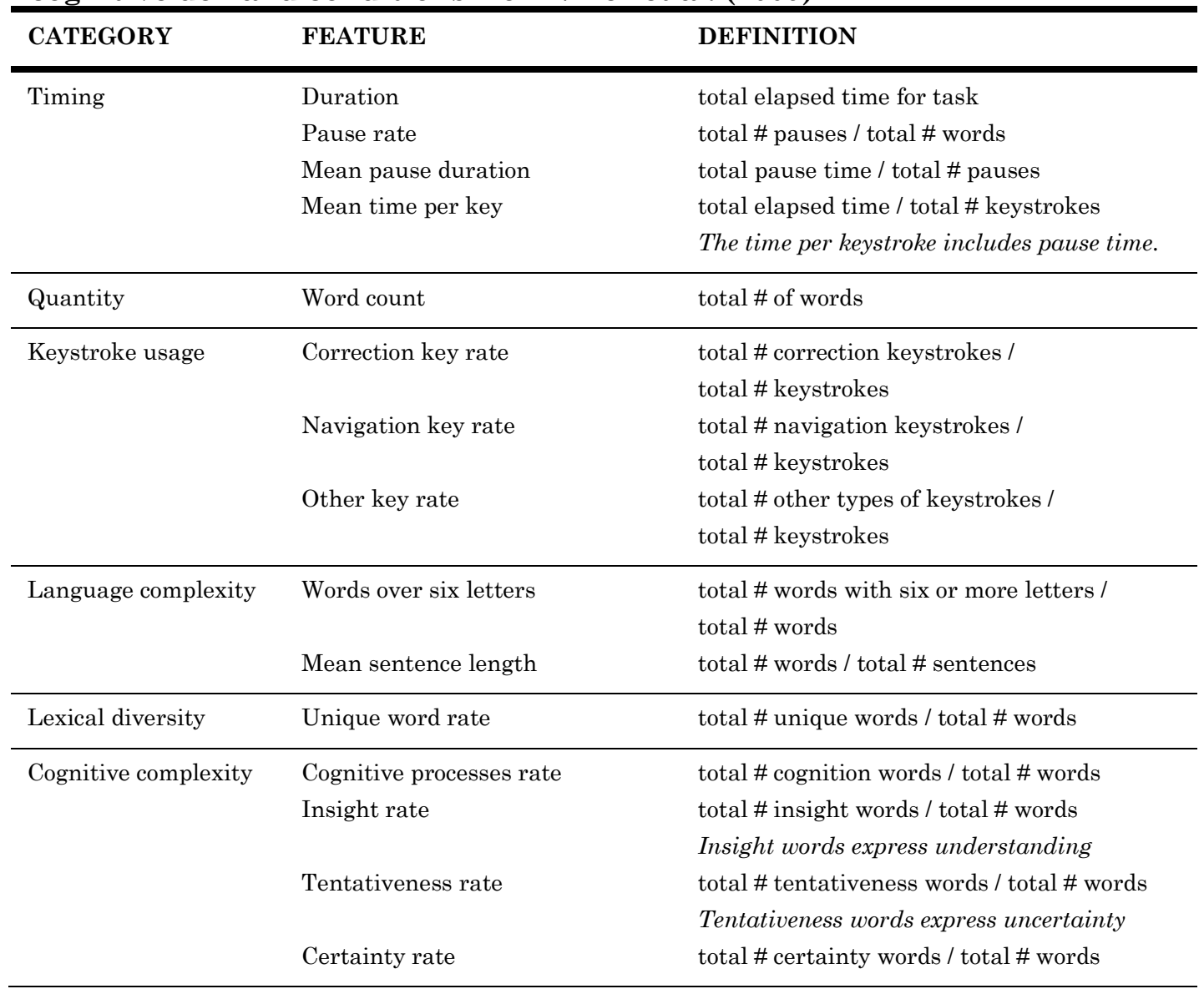




\begin{tabular}{lll}
\hline & & Certainty words express conviction. \\
\hline Affect & $\begin{array}{l}\text { Negative affect rate } \\
\text { Positive affect rate }\end{array}$ & $\begin{array}{l}\text { total \# positive affect words / total \# words } \\
\text { total \# negative affect words / total \# words }\end{array}$ \\
\hline Personal pronouns & Third-person singular pronouns & $\begin{array}{l}\text { total \# third-person singular pronouns / } \\
\text { total \# words } \\
\text { total \# third-person plural pronouns / } \\
\end{array}$ \\
& Third-person plural pronouns & total \# words \\
\hline Sensing & Perception word rate & $\begin{array}{l}\text { total \# sensory words / total \# words } \\
\text { Sensory words express sensorial experiences } \\
\text { such as seeing, hearing, and feeling. }\end{array}$ \\
&
\end{tabular}

In a related study, Epp et al. (2011) used keystroke dynamics features to detect emotional states from free-text typing samples. The models employed means and standard deviations of keystroke durations (time per key), latencies, and events for discriminating among 15 emotional states. Models classified seven emotional states with accuracy rates over $75 \%$. However, the method did not detect stress at this high rate and the classification accuracy was not reported. They did not incorporate language features.

Feild et al. (2010) studied features of search tasks to detect searcher frustration. Using character and token length of queries, duration of task, URL count for task, and actions per task they reached $\mathrm{F}(\beta=0.5)=0.80$. This model is very specific to the type of task studied and may not generalize to other tasks, requiring further study.

More recently, Hernandez et al. (2014) tested whether they could sense stress conditions using a pressure-sensitive keyboard and capacitive mouse. Correct classification rates were $>79 \%$ for the keyboard and $75 \%$ for the mouse. However, this approach requires specialized equipment.

The study in this paper focuses on text-based keyboard interactions only. We made this decision to simplify the experimental design and use only readily available equipment.

\subsubsection{Timing, language, and cognitive demand}

We defined high cognitive demand as an amount of cognitive stress and cognitive load that an individual experiences as taxing or overwhelming. Given this definition, we can compile the keystroke and language features predicted to change under both high cognitive load and high cognitive stress to produce a larger set of features predicted to change under high cognitive demand. The summary list is shown in Table IV along with the body of literature from which each feature originated. Note that the Cognitive processes rate is a composite variable calculated by LIWC from Insight and Certainty (in Table IV) as well as measures of Tentativeness, Causation, Discrepancy, and Differentiation.

Table IV. Timing and language features predicted to change under high cognitive demand conditions by cognitive load and stress literatures with inferred keyboard interaction features predicted to change.

\begin{tabular}{llccc}
\hline \multirow{2}{*}{ CATEGORY } & FEATURE & LOAD & STRESS & KNTEYBOARD \\
\hline \multirow{2}{*}{ Timing } & Duration & $\checkmark 4$ & $\checkmark^{7}$ & \\
& Pause rate & $\checkmark^{4}$ & $\checkmark^{1}$ & $\checkmark 6$ \\
\hline
\end{tabular}




\begin{tabular}{|c|c|c|c|c|}
\hline & Mean pause duration & $\checkmark^{4}$ & $\checkmark 1$ & $\sqrt{6}$ \\
\hline & Mean time per key & & & $\sqrt{6}$ \\
\hline \multirow[t]{3}{*}{ Keystroke usage } & Self-correction rate & & $\sqrt{8}$ & $\sqrt{6}$ \\
\hline & Navigation key rate & & & $\sqrt{6}$ \\
\hline & Other key rate & & & $\sqrt{6}$ \\
\hline \multirow[t]{3}{*}{ Language complexity } & Word count & $\checkmark^{2}$ & $\sqrt{ } 7$ & \\
\hline & Mean sentence length & $\sqrt{ }^{2}$ & $\checkmark 7$ & \\
\hline & Words over six letters & $\sqrt{ }^{2}$ & $\sqrt{7}$ & \\
\hline Lexical diversity & Unique word rate & $\sqrt{2}$ & $\sqrt{7}$ & \\
\hline \multirow[t]{4}{*}{ Cognitive complexity } & Cognitive processes rate & $\sqrt{5}$ & $\sqrt{8}$ & \\
\hline & Insight rate & $\sqrt{6}$ & $\sqrt{6}$ & \\
\hline & Certainty rate & & $\sqrt{7}$ & \\
\hline & Tentativeness rate & $\sqrt{6}$ & $\sqrt{6}$ & \\
\hline Sensing & Perception word rate & $\sqrt{5}$ & & \\
\hline \multirow[t]{2}{*}{ Affect } & Positive emotion rate & $\sqrt{3}$ & $\sqrt{ } 7$ & \\
\hline & Negative emotion rate & $\sqrt{3}$ & $\sqrt{7}$ & \\
\hline \multirow[t]{4}{*}{ Personal pronouns } & First-person singular pronoun rate & $\sqrt{3}$ & $\sqrt{ } 7$ & \\
\hline & Third-person singular pronouns rate & $\sqrt{ } 3$ & & \\
\hline & First-person plural pronouns rate & $\sqrt{3}$ & & \\
\hline & Third-person plural pronouns rate & $\sqrt{3}^{3}$ & & \\
\hline $\begin{array}{l}{ }^{1} \text { DePaulo et al. } 2003 \\
{ }^{2} \text { Khawaja and Chen } 2 \\
{ }^{3} \text { Khawaja et al. } 2013 \\
{ }^{4} \text { Schilperoord } 2002 \\
{ }^{5} \text { Sweller et al. } 2011 \\
{ }^{6} \text { Vizer and Sears } 2009 \\
{ }^{7} \text { Zhou et al. } 2003 \\
{ }^{8} \text { Zhou 2005 }\end{array}$ & & & & \\
\hline
\end{tabular}

\subsection{Summary}

Cognitive functions, including memory and processing, are utilized extensively during interactions with IT, so any factor that impacts cognitive function can impact performance on tasks involving IT. The importance of monitoring cognitive function is becoming increasingly clear as we learn more about the impact of daily stress on cognition, physical health, and mental health. More effective monitoring is needed, and there are several opportunities for improvement over current methods.

Existing solutions are inadequate for frequent and convenient monitoring of the status of cognitive function. Current assessments from the human-centered computing community, such as affective computing, typically only measure overt and predictable reactions to acute stress during the course of the event, and they can be obtrusive, require supplementary equipment, and are often labor or computationally intensive. Insights from keystroke dynamics and language analysis research illustrate the possibility of leveraging everyday keyboard interactions for unobtrusive monitoring of cognitive function. Real-time monitoring of keyboard activity allows analysis of paralinguistic cues such as input rate, pause activity, and error and correction activity (Zuckerman et al. 1981), cues we cannot analyze with linguistic 
analysis alone. Over time, changes in salient keystroke and linguistic features could indicate changes in cognitive function due to high cognitive load and stress.

In an exploratory study of this concept, Vizer et al. (Vizer et al. 2009) established that changes in keystroke and linguistic features indicated presence of cognitive demand for a group of participants, but did not examine the cognitive demand response on an individual level. This research will briefly reexamine the generic analysis and compare those results to the individual cognitive demand responses of a subset of participants.

\section{METHODS}

This study examined the effects of changes in cognitive demand on timing, keystroke, and linguistic patterns by modeling cognitive demand response for the entire participant group as well as for a subset of individuals.

\subsection{Hypotheses}

The preceding discussion outlined the need for more sensitive, convenient, and frequent assessments to monitor cognitive status, and examined analysis of text input as one possible solution. From this review stem the following hypotheses:

$\mathrm{H} 1_{\mathrm{A}}$. The generic model will classify samples with accuracy significantly better than chance.

H2A. The individuals models will classify samples with accuracy significantly better than chance.

H3A. Individual models will perform better than the generic model.

Hypotheses $\mathrm{H} 1$ and $\mathrm{H} 2$ look at whether the generic and individualized models perform better than the null model at classifying control versus experimental samples. Hypothesis H3 explores whether personalized models are more effective than generic models. We evaluated these hypotheses by analyzing features drawn from keystroke dynamics and language literature for differences between samples produced under low cognitive demand control conditions and those produced under high cognitive demand experimental conditions.

\subsection{Assumptions}

This study makes three assumptions about typing behavior during data collection regarding the performance at baseline and overall performance.

Baseline Performance: This study assumes that the baseline condition simulates a normal low cognitive demand situation. To mitigate risk, a task load questionnaire was used to gauge cognitive demand level.

Typing Performance: This study assumes that typing performance for each participant does not change appreciably over the course of data collection. To mitigate this risk, data were collected over as short a period as was manageable. Cognitive Demand Due to Experiment: This study assumes that any cognitive demand induced by participation in the study itself is negligible and/or constant. To mitigate this risk, the experimental setup was the same for all sessions.

\subsection{Equipment and Software}

Data collection apparatus included an all-in-one desktop computer with standard peripherals. All participants used the same experimental equipment. This computer had a 20.5" flat screen monitor, a full-size standard keyboard, and a full-size standard mouse. Software included Visual Basic programs for collecting typing 
samples and administering the cognitive stress tasks. The first author designed and implemented the software. All software used by participants employed a minimum of 16-pt typeface to ensure readability. This typeface displayed at a letter height of $3.175 \mathrm{~mm}$. Participant viewing distance was $50 \mathrm{~cm}$ to $60 \mathrm{~cm}$.

We used the Symbol Digit Modalities Test (Smith 1982) to confirm normal cognitive function for each participant. Normal is defined as scoring less than -2.0 standard deviations below the age-adjusted mean. This test was selected due to its easy administration and scoring as well as its high sensitivity to cerebral dysfunction from a wide range of causes.

We used the NASA-Task Load Index (NASA-TLX) to assess workload after each typing task. Validity and reliability of this assessment has been established repeatedly (Hart and Staveland 1988). The pair-wise comparisons sometimes used when gathering data with the NASA-TLX were not used in this study. Effects of eliminating the pair-wise comparisons have been studied previously with results validating the efficacy of the tool without these comparisons (Byers et al. 1989; Moroney et al. 1992). Of particular interest in this study are the Mental Demand and Frustration scales. Mental Demand is defined as "How much mental and perceptual activity was required (e.g., thinking, deciding, calculating, remembering, looking, searching, etc.)? Was the task easy or demanding, simple or complex, exacting or forgiving? (Hart and Staveland 1988)" Frustration is defined as "How insecure, discouraged, irritated, stressed and annoyed versus secure, gratified, content, relaxed and complacent did you feel during the task? (Hart and Staveland 1988)" Mental Demand is a measure of cognitive load and Frustration is a proxy for cognitive stress.

Participants also completed a demographic survey including questions on age, educational level, computer experience, and self-rated typing skill.

\subsection{Experimental Design}

This section describes the experiments investigating effects of cognitive stress on typing patterns.

\subsubsection{Participants}

The study included 20 younger adults recruited from a mid-size mid-Atlantic public university. The mean (SD) age was 24 (4.5) years of age. Eleven participants were female and 18 were right-handed. Participants reported a mean (SD) of 10.2 (4.5) years of typing experience and had a self-rated typing proficiency of slightly better than average on a scale from -2 to +2 , with 0 being average (mean=0.2, $\mathrm{SD}=0.6$ ). All participants had at least some college education, vision corrected to better than $20 / 60$, at least one year of typing experience, no cognitive impairments, and were free of physical impairments that might adversely affect the use of a keyboard or mouse. Cognitive function was assessed using the Symbol Digit Modalities Test (Smith 1982) and all participants scored in the normal range. We assessed cognitive function to reduce the possibility that differences between control and experimental samples were due to cognitive-related causes other than demand. Physical function was assessed through observation. Experimental sessions for each participant took place at the university. Participants were compensated $\$ 40$ for the baseline protocol and an additional $\$ 80$ for the experimental protocol.

\subsubsection{Baseline Protocol}

The baseline condition was composed of four data collection sessions each lasting a total of 20-45 minutes, depending on the participant's typing speed. Participants completed three typing samples under low cognitive demand conditions during each session for a total of 12 baseline samples per participant. Individual sessions were 
separated by at least two hours with no more than two sessions in one day. During the first baseline session each participant completed the consent procedure and the demographic survey. This process added about 10-15 minutes to the length of the first session.

During each baseline session, each participant produced three samples of spontaneously generated (free) text by typing to fill a text box that held approximately 700-800 characters. The length of the free text is based upon results of an earlier study that found a sample of this size produced a sufficient quantity of text for analysis (Gunetti and Picardi 2005). Participants were free to type on any topic they selected. We provided prompts that could be used as motivation for the text if the participant desired (ex., "Family," "Hobbies," Your weekend activities"). We intended this condition to simulate real-world low cognitive demand composition conditions as closely as possible. After each typing sample, participants completed the NASA-TLX. Participants were permitted to rest between samples if desired.

\subsubsection{Experimental Protocol}

Experimental sessions were scheduled after the participant completed all baseline sessions. Individual sessions were separated by at least two hours with no more than two sessions in one day. Participants produced two control samples and two experimental samples per experimental session. All 20 participants completed two basic experimental sessions, providing a total of four control and four experimental samples each. All participants were offered the opportunity to complete the extended protocol of 13 additional sessions, and a subset of nine participants accepted, providing a total of an additional 26 control and experimental samples each. The larger data set allows for analysis of samples on an individual level. Each experimental data collection session lasted approximately 45-60 minutes. Details of the samples collected during the protocols are in Table V.

Table V. Experimental design per participant

\begin{tabular}{lcll}
\hline PROTOCOL & SESSIONS & SAMPLES/SESSION & TOTAL SAMPLES \\
\hline Baseline & 4 & 3 baseline & 12 baseline \\
Basic & 2 & 2 control, & 4 control, \\
Experimental & & 2 experimental & 4 experimental \\
Extended & \multirow{2}{*}{13} & 2 control, & 26 control, \\
Experimental & & 2 experimental & 26 experimental \\
\hline
\end{tabular}

The control condition mimicked the low cognitive demand baseline condition. At the beginning of each experimental session the participant provided two control samples under the same conditions as the baseline samples as presented in Section 3.4.2.

The experimental condition featured a combined task of first a high cognitive demand task then provision of a typing sample. The participant completed this combined task twice. For the high cognitive demand task we employed n-back, or Lag-n, number recall (Dobbs and Rule 1989; Owen et al. 2005). This task is widely used to induce high cognitive load. N-back number recall requires the participant to respond with the number $n$ back from the last stimulus. For example, if $n=3$ and the last three stimuli were " 3 ", " 4 ", and " 5 ", the participant would respond with " 3 " after the " 5 " was presented. This task requires that $\mathrm{n}$ numbers be kept in short-term memory at all times. Common variants are one-back, two-back, or three-back tests. A four-back test has been found to produce too much load to be useful (Owen et al. 2005). 
We chose the three-back variant for this study. Participants performed the task without the aid of external cognition such as paper and pencil.

For this task, the stimuli began as a one-digit number. After 20 correct answers, the number of digits in the stimulus increased by one. Participants had two opportunities to provide the correct answer before being presented with the next stimulus. Each task continued until the participant gave twenty incorrect answers at a given level of difficulty. Upon completion of the high cognitive demand task the participant was presented with the same text box as used for the baseline and control conditions.

We used a pretest-posttest design to ensure that first-order carry-over effects, such as cognitive fatigue from the high cognitive demand conditions, did not affect the control samples, thereby negatively impacting the correspondence to baseline samples. The same length and content parameters applied to the control and experimental typing samples as applied to the baseline typing samples. After each typing sample, participants completed the NASA-TLX. For the control conditions the participant assessed the workload for just the typing sample. For the experimental conditions, the participant assessed the workload for the combined high cognitive demand and typing task.

\subsection{Data Analysis}

Typing samples were analyzed to establish models for each condition.

\subsubsection{Keystroke and Linguistic Features}

Each typing sample consists of spontaneously generated free text of approximately 700-800 characters. The data collected during the typing task consists of the type of keyboard event (either key up or key down), time stamp, and key code for each keyboard event. Experiment software recorded the time stamp to a resolution of 10 milliseconds using the Ticks call to the system clock in Visual Basic. Key codes allow for analysis of the keys pressed and words typed. The software saved the raw text at the completion of the typing task.

We extracted the keystroke and linguistic features in Table VI from the raw keystroke and text data for each text sample. We used Python Natural Language Toolkit (Bird et al. 2009), Linguistic Inquiry and Word Count (LIWC) (Pennebaker et al. 2001), and custom software for feature extraction. Specific features were selected from research on free text keystroke analysis, language under cognitive load, and text-based deception-detection (Mehrabian and Wiener 1966; Villani et al. 2006; Zhou 2005; Zhou, Burgoon, Nunamaker, et al. 2004; Zhou and Zhang 2006; Khawaja et al. 2013; Khawaja and Chen 2010; Yin et al. 2007), most of which were used in a prior study of cognitive demand and typing patterns (Vizer et al. 2009). Note that Cognitive processes rate is a composite variable calculated by LIWC from Insight, Certainty, and Tentativeness (in Table VI) as well as measures of Causation, Discrepancy, and Differentiation. For comparisons across individuals, features from the control and experimental samples were normalized to z-scores using means and standard deviations calculated per feature from the baseline samples. For comparisons within individuals, we analyzed raw data, as each participant served as his or her own control.

Table VI. Keystroke and language features extracted from typing samples

\begin{tabular}{llll}
\hline CATEGORY & FEATURE & DEFINITION & EXAMPLE \\
\hline Timing & Duration & Total elapsed time for typing task & \\
\hline
\end{tabular}




\begin{tabular}{|c|c|c|c|}
\hline & Pause rate & $\begin{array}{l}\text { total \# pauses / total \# keystrokes } \\
\text { Pauses are defined as instances of no } \\
\text { keyboard activity for over } 1 / 2 \text { sec. }\end{array}$ & \\
\hline & Mean pause duration & total pause time / total \# pauses & \\
\hline & Mean time per key & total elapsed time / total \# keystrokes & \\
\hline & & This time per keystroke includes pause time. & \\
\hline & $\begin{array}{l}\text { Adjusted mean time } \\
\text { per key }\end{array}$ & $\begin{array}{l}\text { (total elapsed time - total pause time) / } \\
\text { total \# keystrokes }\end{array}$ & \\
\hline & & $\begin{array}{l}\text { This time per keystroke does not include } \\
\text { pause time. }\end{array}$ & \\
\hline \multirow{3}{*}{$\begin{array}{l}\text { Language } \\
\text { complexity }\end{array}$} & Word count & total \# words & \\
\hline & Mean sentence length & total \# words / total \# sentences & \\
\hline & Words over six letters & $\begin{array}{l}\text { total \# words with six or more letters / } \\
\text { total \# words }\end{array}$ & \\
\hline \multirow[t]{4}{*}{$\begin{array}{l}\text { Keystroke } \\
\text { usage }\end{array}$} & Correction key rate & $\begin{array}{l}\text { total \# correction keystrokes / total \# } \\
\text { keystrokes }\end{array}$ & $\begin{array}{l}\text { Backspace, } \\
\text { delete, home, } \\
\text { end, arrow }\end{array}$ \\
\hline & Function key rate & $\begin{array}{l}\text { total \# function keystrokes / total \# } \\
\text { keystrokes }\end{array}$ & $\begin{array}{l}\text { Control, tab, } \\
\text { alt }\end{array}$ \\
\hline & Other key rate & total \# other keystrokes / total \# keystrokes & “m”, “6”, “p” \\
\hline & & $\begin{array}{l}\text { "Other" keys are those not counted in any of } \\
\text { the above rates, such as letter and number } \\
\text { keys. }\end{array}$ & \\
\hline $\begin{array}{l}\text { Lexical } \\
\text { diversity }\end{array}$ & Lexical diversity & total \# unique words / total \# words & \\
\hline \multirow{7}{*}{$\begin{array}{l}\text { Cognitive } \\
\text { complexity }\end{array}$} & Cognitive processes & total \# cognition words / total \# words & "cause", \\
\hline & & $\begin{array}{l}\text { This is a composite variable calculated by } \\
\text { LIWC including Insight, Certainty, and } \\
\text { Tentativeness (below) as well as Causation, } \\
\text { Discrepancy, and Differentiation. }\end{array}$ & "remember" \\
\hline & Insight rate & total \# insight words / total \# words & "think", \\
\hline & & Insight words express understanding & know \\
\hline & Certainty rate & total \# certainty words / total \# words & "always", \\
\hline & & Certainty words express conviction. & \\
\hline & Tentativeness rate & $\begin{array}{l}\text { total \# tentativeness words / total \# words } \\
\text { Tentativeness words express uncertainty. }\end{array}$ & $\begin{array}{l}\text { "maybe", } \\
\text { "perhaps" }\end{array}$ \\
\hline \multirow[t]{2}{*}{ Sensing } & Perception word rate & total \# sensory words / total \# words & "taste", \\
\hline & & $\begin{array}{l}\text { Sensory words express sensorial experiences } \\
\text { such as seeing, hearing, and feeling. }\end{array}$ & "hear" \\
\hline \multirow[t]{2}{*}{ Affect } & Positive emotion rate & total \# positive affect words / total \# words & "like", "calm" \\
\hline & & $\begin{array}{l}\text { Positive affect is defined as conscious } \\
\text { subjective aspect of a positive emotion apart }\end{array}$ & \\
\hline
\end{tabular}




\begin{tabular}{|c|c|c|c|}
\hline \multicolumn{4}{|c|}{ from bodily changes. } \\
\hline & Negative emotion rate & total \# negative affect words / total \# words & "hate", "fear" \\
\hline & & $\begin{array}{l}\text { Negative affect is defined as conscious } \\
\text { subjective aspect of a negative emotion apart } \\
\text { from bodily changes. }\end{array}$ & \\
\hline \multirow[t]{2}{*}{$\begin{array}{l}\text { Personal } \\
\text { pronouns }\end{array}$} & $\begin{array}{l}\text { First-person pronoun } \\
\text { rate }\end{array}$ & total \# first-person pronouns / total \# words & $\begin{array}{l}\text { "I", "mine", } \\
\text { "us", "our" }\end{array}$ \\
\hline & $\begin{array}{l}\text { Third-person pronoun } \\
\text { rate }\end{array}$ & total \# third-person pronouns / total \# words & $\begin{array}{l}\text { "he", "hers", } \\
\text { "they" }\end{array}$ \\
\hline
\end{tabular}

\subsubsection{Classification Models}

We tested the hypotheses using a statistical method to build classification models. We evaluated the classification models to determine their accuracy in discriminating typing samples from control and experimental conditions.

Using binary logistic regression (Menard 2002), we built and tested classification models for discrimination between control and experimental samples. We used a cutoff value of $p=0.5$ for classification. Logistic regression is appropriate for discrimination between two groups, is robust to non-normally distributed variables, and Zhou et al. (Zhou, Burgoon, Twitchell, et al. 2004) used it successfully for classifying typing samples for deception detection.

To build the generic model we used the 80 basic experimental protocol samples from all participants and to build each individual model we used 30 samples that include the participant's four basic protocol samples plus the 26 extended protocol samples. We employed the wrapper method of scheme-independent variable selection (Witten et al. 2011) to define the variable subset used in each model. We performed the regression using a cutoff of $p=0.25$ for entry and removal (Hosmer Jr et al. 2013) for forward selection of variables. We kept the sample-to-variable ratio threshold to greater than 10 (Jain et al. 2000), resulting in no more than 16 variables allowed in the generic model and no more than six in each individual model. If a regression resulted in a model with too many variables, we rolled back to the last forward selection step with an acceptable sample-to-variable ratio. After we selected a candidate variable set, we tested models for goodness-of-fit using classification accuracy, $\chi^{2}$, and the Area Under the Curve (AUC) for the Receiver Operating Characteristic (ROC) analysis.

We evaluated the performance of each statistical model using leave-one-out (LOO) cross-validation (Witten et al. 2011) to predict the error rate. For the generic model, we trained the model using the data from 19 participants to calculate the predicted probabilities for the 20th participant's samples. We repeated this procedure for all participants and report the mean classification accuracy across all participants. For the individual models, we trained each model using 29 samples from the participant to calculate the predicted probability for the 30th sample. We repeated this procedure for all samples and report the mean classification accuracy across all samples per participant.

\section{RESULTS}

The first section reports on the significant differences in cognitive demand between low and high cognitive demand conditions as measured by the NASA-TLX subscales. Next, we describe the generic and personalized classification models built from the feature sets. Lastly, a comparison of results of the generic and personalized models is shown. This analysis expands on a prior paper (Vizer 2013) that provided only a 
short summary of the personalized model results. Details of the data collected per protocol are in Table VII.

Table VII. Data collected per protocol

\begin{tabular}{lccll}
\hline PROTOCOL & PARTICIPANTS & SESSIONS & SAMPLES/SESSION & TOTAL SAMPLES \\
\hline Baseline & 20 & 4 & 3 baseline & 240 baseline \\
Basic & 20 & 2 & 2 control, & 80 control, \\
Experimental & \multirow{2}{*}{13} & 2 experimental & 80 experimental \\
Extended & 9 & 2 experimental & 234 control, \\
Experimental & & & 232 experimental* \\
\hline * Participant one missed two experimental samples & & \\
\hline
\end{tabular}

Results confirm that participants did report higher cognitive load and cognitive stress during the experimental conditions. The goodness-of-fit statistics and crossvalidation analysis showed that the generic model is a significant improvement over the null model. Individual models also show a better fit to the data than the null models. Furthermore, the personalized individual models performed significantly better than the generic model.

\subsection{Comparison of self-reported cognitive load and cognitive stress under control and experimental conditions}

We obtained NASA-TLX ratings for each control and experimental sample and conducted paired sample t-tests on the Mental Demand and Frustration subscales. By definition these subscales are measures of cognitive load and cognitive stress, the two components of cognitive demand. Descriptive statistics are in Table VIII. As the data are not normally distributed, the non-parametric Wilcoxon signed-rank test was employed. For the generic analysis including all participants, the Wilcoxon signedrank test determined that ratings for were significantly higher for the experimental conditions versus the control conditions with $p<0.001$ for both subscales. This confirms that, overall, participants experienced significantly higher cognitive load and stress levels during the experimental protocol. However, it is important to note that although all individual participants reported an increase in cognitive load and stress under experimental conditions, they did exhibit differences in magnitude of change in experienced load and stress. For the individual participants in the extended protocol, eight out of nine participants showed statistically significant differences $(p<0.05)$ for each of the two subscales.

Table VIII. Results of Wilcoxon signed-rank test comparing control and experimental NASA-TLX Mental Demand and Frustration subscales

MENTAL DEMAND

(COGNITIVE LOAD)
FRUSTRATION (COGNITIVE STRESS)

\begin{tabular}{ccccccccccc} 
Model & \multicolumn{2}{c}{$\begin{array}{c}\text { Control } \\
\text { Mean (SD) }\end{array}$} & \multicolumn{2}{c}{$\begin{array}{c}\text { Experimental } \\
\text { Mean (SD) }\end{array}$} & $\boldsymbol{Z}$ & \multicolumn{2}{c}{$\begin{array}{c}\text { Control } \\
\text { Mean (SD) }\end{array}$} & \multicolumn{2}{c}{$\begin{array}{c}\text { Experimental } \\
\text { Mean (SD) }\end{array}$} & $\boldsymbol{Z}$ \\
\hline Generic & 35.2 & $(28.4)$ & 57.4 & $(27.0)$ & $-4.609^{* * *}$ & 20.0 & $(21.8)$ & 35.9 & $(26.3)$ & $-3.731^{* * *}$ \\
\hline P1 & 31.9 & $(11.2)$ & 47.4 & $(8.6)$ & $-4.251^{* * *}$ & 7.1 & $(0.98)$ & 11.6 & $(1.6)$ & $-2.337^{*}$ \\
\hline P2 & 18.3 & $(10.2)$ & 22.0 & $(18.2)$ & -0.853 & 15.0 & $(19.7)$ & 16.8 & $(16.7)$ & -1.388 \\
\hline P3 & 61.3 & $(12.4)$ & 68.7 & $(9.6)$ & $-3.763^{* * *}$ & 7.7 & $(3.7)$ & 14.7 & $(6.4)$ & $-4.257^{* * *}$ \\
\hline P4 & 7.2 & $(6.8)$ & 56.5 & $(11.8)$ & $-4.794^{* * *}$ & 3.0 & $(4.5)$ & 23.8 & $(16.5)$ & $-4.723^{* * *}$ \\
\hline P5 & 4.7 & $(3.5)$ & 77.8 & $(7.2)$ & $-4.822^{* * *}$ & 2.0 & $(3.1)$ & 19.8 & $(17.2)$ & $-4.811^{* * *}$ \\
\hline
\end{tabular}




\begin{tabular}{rrrrrrrrrrr}
\hline P6 & 30.3 & $(9.9)$ & 46.8 & $(13.5)$ & $-4.578^{* * *}$ & 29.8 & $(9.5)$ & 41.8 & $(10.3)$ & $-4.578^{* * *}$ \\
\hline P7 & 50.3 & $(5.1)$ & 73.8 & $(7.3)$ & $-4.734^{* * *}$ & 1.3 & $(2.2)$ & 13.8 & $(4.9)$ & $-4.858^{* * *}$ \\
\hline P8 & 50.3 & $(7.2)$ & 60.7 & $(4.9)$ & $-4.501^{* * *}$ & 48.7 & $(11.7)$ & 65.7 & $(6.7)$ & $-4.644^{* * *}$ \\
\hline P9 & 33.8 & $(51.6)$ & 37.8 & $(12.2)$ & $-4.130^{* * *}$ & 29.5 & $(18.8)$ & 50.0 & $(13.3)$ & $-4.399^{* * *}$ \\
\hline
\end{tabular}

Note: ${ }^{*} \mathrm{p}<0.05,{ }^{* *} \mathrm{p}<0.01,{ }^{* * *} \mathrm{p}<0.001$

\subsection{Comparison of generic and individual cognitive demand classification models}

Descriptive statistics for the variables in the generic analysis and t-tests between conditions are in Appendix A. Descriptive statistics for the variables and t-tests between conditions for each individual analysis are in Appendix B. The significance level for $p$ was adjusted to 0.0025 using the Bonferroni correction for multiple comparisons.

Using the subset selection process discussed in the Methods section, we selected the variables in Table IX as predictors for cognitive demand for the generic and individual analyses. We then performed logistic regression to determine the variable coefficients and standard errors for each variable in each model. Models for P2 and $\mathrm{P} 6$ resulted in more variables than acceptable for an appropriate sample-to-feature ratio so each model was rolled back to the last step of the forward selection process with an acceptable number of variables.

Classification accuracy and goodness-of-fit measures for the generic and individual models are also displayed in Table IX. Using stratified LOO crossvalidation the classification accuracy was $66.9 \%$ for the generic model. This represents an improvement over the chance rate of correct classification of $50 \%$. We also calculated $\chi^{2}$, which indicates that the new model is a significantly better fit to the data than the null model.

For the personalized models, classification accuracy ranged from $65.0 \%$ to $93.3 \%$ (Mean $=78.1 \%, \mathrm{SD}=8.91$ ). The chance classification rate for each individual is $50 \%$, except for Participant 1 whose chance rate was $51.7 \%$ due to two missed experimental samples. The AUCs ranged from 0.679 to 0.953 . The $\chi^{2}$ statistic is also listed for each participant's model. All participants show significant goodness-of-fit as indicated by at least one of the AUC or $\chi^{2}$ statistics. These results indicate that models were a better fit to the data than the null model.

Table IX. Logistic regression models for generic and individual analyses

\begin{tabular}{|c|c|c|c|c|}
\hline \multirow[b]{2}{*}{ MODEL STATISTICS } & \multirow[b]{2}{*}{ VARIABLES IN MODEL } & \multirow[b]{2}{*}{ ODDS RATIO } & \multicolumn{2}{|c|}{$95 \% \mathrm{CI}$} \\
\hline & & & UPPER & LOWER \\
\hline GENERIC & Word count & 0.823 & 0.638 & 1.061 \\
\hline 0.669 & Words per sentence* & 0.738 & 0.549 & 0.992 \\
\hline AUC $\quad 0.714 * * *$ & Words over six letters & 0.809 & 0.577 & 1.135 \\
\hline$\chi^{2} \quad 24.867 * *$ & Negative emotion* & 1.233 & 1.005 & 1.512 \\
\hline & Duration & 0.539 & 0.287 & 1.013 \\
\hline & Pause Count* & 6.731 & 2.034 & 22.279 \\
\hline & Time per keystroke & 1.567 & 0.859 & 2.857 \\
\hline & Adjusted time per keystroke & 1.220 & 0.917 & 1.623 \\
\hline & Pause rate* & 0.142 & 0.038 & 0.530 \\
\hline & Other key rate & 1.265 & 0.916 & 1.746 \\
\hline
\end{tabular}




\begin{tabular}{|c|c|c|c|c|c|c|}
\hline & & & & & & \\
\hline & & & Constant & 0.979 & & \\
\hline \multirow[t]{4}{*}{$\mathbf{P} 1$} & & & Words per sentence* & 1.143 & 1.028 & 1.271 \\
\hline & CA & 0.847 & Negative emotion** & 2.501 & 1.399 & 4.471 \\
\hline & AUC & $0.889 * * *$ & Lexical diversity* & 1.499 & 1.047 & 2.146 \\
\hline & $\chi^{2}$ & $29.714 * * *$ & Constant ${ }^{* * *}$ & 0.000 & & \\
\hline \multirow[t]{6}{*}{ P2 } & & & Negative emotion* & 0.502 & 0.274 & 0.922 \\
\hline & CA & 0.883 & Lexical diversity*** & 1.694 & 1.230 & 2.334 \\
\hline & AUC & $0.919 * * *$ & Tentativeness & 1.746 & 0.935 & 3.260 \\
\hline & $\chi^{2}$ & $36.597 * * *$ & Other key rate & $4.475 \mathrm{E}+13$ & 0.043 & $4.698 \mathrm{E}+28$ \\
\hline & & & Duration** & 1.000 & 1.000 & 1.000 \\
\hline & & & Constant* & 0.000 & & \\
\hline \multirow[t]{5}{*}{ P3 } & & & Negative emotion & 1.355 & 0.975 & 1.882 \\
\hline & CA & 0.750 & Tentativeness * & 1.499 & 1.032 & 2.179 \\
\hline & AUC & $0.771 * *$ & Words over six letters & 0.854 & 0.727 & 1.004 \\
\hline & $\chi^{2}$ & $16.288 * *$ & Time per keystroke* & 0.000 & 0.000 & 0.029 \\
\hline & & & Constant* & 101339.023 & & \\
\hline \multirow[t]{7}{*}{$\mathbf{P} 4$} & & & Negative Emotion** & 4.983 & 1.689 & 14.701 \\
\hline & CA & 0.933 & Words over six letters** & 0.616 & 0.435 & 0.873 \\
\hline & AUC & $0.953 * * *$ & Time per keystroke & $1.636 \mathrm{E}+33$ & 0.000 & $1.359 \mathrm{E}+87$ \\
\hline & $\chi^{2}$ & $49.277 * * *$ & Third person pronouns ${ }^{* *}$ & 0.369 & 0.174 & 0.781 \\
\hline & & & Insight* & 2.525 & 1.209 & 5.274 \\
\hline & & & Correction key rate* & $2.123 \mathrm{E}+80$ & $2.159 \mathrm{E}+17$ & $2.088 \mathrm{E}+143$ \\
\hline & & & Constant & 0.000 & & \\
\hline \multirow[t]{7}{*}{ P5 } & & & Words over six letters & 1.287 & 0.997 & 1.662 \\
\hline & CA & 0.750 & Correction key rate * & $1.003 \mathrm{E}+34$ & 38855.481 & $2.3321 \mathrm{E}+64$ \\
\hline & AUC & $0.852 * * *$ & Dictionary words* & 1.435 & 1.001 & 2.057 \\
\hline & $\chi^{2}$ & $27.324 * * *$ & Cognitive processes rate ${ }^{* *}$ & 0.722 & 0.579 & 0.901 \\
\hline & & & Certainty & 0.465 & 0.199 & 1.089 \\
\hline & & & Perceptual processes rate & 1.621 & 0.959 & 2.741 \\
\hline & & & Constant & 0.000 & & \\
\hline \multirow[t]{7}{*}{$\mathbf{P 6}$} & & & Correction key rate & $3.611 \mathrm{E}+10$ & 0.000 & $7.758 \mathrm{E}+25$ \\
\hline & CA & 0.717 & Dictionary words & 0.885 & 0.766 & 1.022 \\
\hline & AUC & $0.791 * * *$ & Cognitive processes rate $*$ & 1.302 & 1.053 & 1.610 \\
\hline & $\chi^{2}$ & $18.486 * *$ & Word count* & 1.075 & 1.003 & 1.152 \\
\hline & & & Positive emotion & 1.377 & 0.880 & 2.153 \\
\hline & & & Negative emotion* & 2.447 & 1.206 & 4.965 \\
\hline & & & Constant & 0.000 & & \\
\hline \multirow[t]{5}{*}{ P7 } & & & Third person pronouns * & 0.540 & 0.307 & 0.952 \\
\hline & CA & 0.767 & Negative emotion & 1.484 & 0.933 & 2.360 \\
\hline & AUC & $0.807 * * *$ & Perceptual processes rate & 0.494 & 0.227 & 1.076 \\
\hline & $\chi^{2}$ & $20.102 * *$ & Pause count** & 0.915 & 0.856 & 0.978 \\
\hline & & & Lexical diversity & 0.791 & 0.589 & 1.062 \\
\hline
\end{tabular}




\begin{tabular}{|c|c|c|c|c|c|c|}
\hline & & & & & & \\
\hline & & & Constant* & 6727.957 & & \\
\hline \multirow[t]{4}{*}{ P8 } & & & Word count & 1.036 & 0.979 & 1.097 \\
\hline & CA & 0.650 & Words per sentence & 0.827 & 0.643 & 1.065 \\
\hline & AUC & 0.679 * & Insight & 1.304 & 0.833 & 2.042 \\
\hline & $\chi^{2}$ & 5.330 & Constant & 0.036 & & \\
\hline \multirow[t]{6}{*}{ P9 } & & & Word count* & 0.857 & 0.740 & 0.991 \\
\hline & CA & 0.733 & Function key rate* & 0.000 & 0.000 & 0.000 \\
\hline & AUC & $0.773 * *$ & Words over six letters & 0.836 & 0.643 & 1.086 \\
\hline & $\chi^{2}$ & $16.187 * *$ & Pause count & 0.889 & 0.790 & 1.001 \\
\hline & & & Lexical diversity* & 1.423 & 1.018 & 1.988 \\
\hline & & & Constant & 0.000 & & \\
\hline
\end{tabular}

Notes: $C A=$ Classification Accuracy, AUC=Area Under Curve, $C I=$ Confidence Interval, ${ }^{*} p<0.05,{ }^{* *} p<0.01,{ }^{* * *} p<0.001$

The AUC and $\chi^{2}$ statistics as well as cross-validation analyses showed that the generic model is an improvement over the chance correct classification rate of $50 \%$ and goodness-of-fit is significantly better than that of the null model. Therefore, we reject the null hypothesis in favor of the alternative hypothesis $\mathrm{H} 1_{\mathrm{A}}$.

All of the individual models show significant goodness-of-fit on at least one of the AUC or $\chi^{2}$ statistics. These results indicate that participants' individual models were a better fit to the data than their null models. Therefore, we reject the null hypothesis in favor of the alternative hypothesis $\mathrm{H} 2 \mathrm{~A}$.

All but one of the individual models produced better classification accuracies than the generic model. The AUCs of the individual models compared to the generic model are significantly better, tested using a one-tailed one-sample t-test ( $p=0.002)$. This shows that the personalized individual models performed significantly better than the generic model. Therefore, we reject the null hypothesis in favor of the alternative hypothesis $\mathrm{H} 3 \mathrm{~A}$.

\section{DISCUSSION}

To put our results in context, we look at the results of other studies on cognitive demand assessment. Affective computing research addressing stress assessment includes automatic optical recognition of facial expressions (Dinges et al. 2007), automatic speech analysis (Yin et al. 2007), instrumented vehicles (Healey and Picard 2000), and pressure-sensing keyboards and mice to detect frustration (Hernandez et al. 2014, Qi et al. 2001). Dinges et al. reported accuracy for automatic optical facial expression recognition to be $68 \%$ when classifying stress versus non-stress conditions (Dinges et al. 2007). Yin et al. obtained a correct classification rate of $71 \%$ from speech analysis on data collected during tasks that induced mental stress (Yin et al. 2007). Data from a car outfitted with electromyogram, electrocardiogram, galvanic skin response, and respiration sensors achieved a correct recognition rate of $88.6 \%$ using an optimal set of features (Healey and Picard 2000). Pressure sensing keyboard data resulted in an accuracy of $>79 \%$ during stressful tasks (Hernandez et al. 2014). Analysis of pressure applied to a computer mouse produced an accuracy of $88 \%$ (Qi et al. 2001). While our results from the generic model analysis are lower than those from the preceding work, the results from the individual model analyses are similar and our method does not require special equipment. This outcome encourages further investigation. 
The wide range of classification accuracies obtained indicates individual differences in the stress response and has implications for systems to monitor for high cognitive demand. One explanation for this phenomenon is that for this task the cognitive demand response curves were different for different people, resulting in larger or smaller differences in y-values for features. These results illustrate important individual differences in the cognitive demand response and are consistent with those from Carneiro et al. (2012), which also showed wide individual differences in the response to cognitive stress. These individual differences are also consistent with Hancock's model of adaptability under stress (Hancock 1989). The highly variable demand response implies that systems that monitor for high cognitive demand must adapt to the individual demand response pattern of each person. The generic model provides a good foundation for features that are likely to show change, but the individual models exhibit a larger range of features that are important to each person's demand response pattern (Figure I). The features figuring most prominently into the individual models in this study are negative emotion, lexical diversity, words over six letters, and word count. All of these except lexical diversity were also included in the generic model. Further research into how to develop an individual's model over time might start with the features from the generic model plus Lexical diversity then use other features to fine tune.

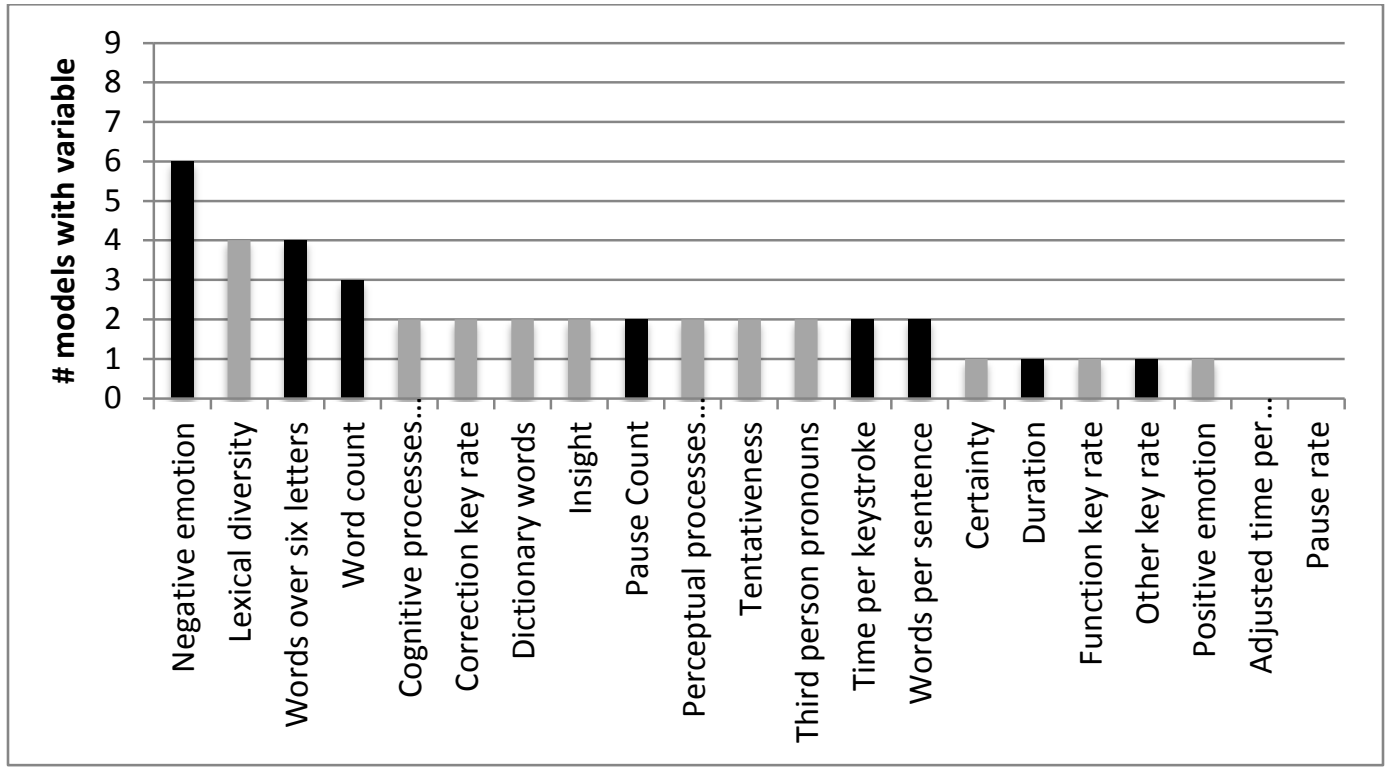

Figure I: Number of individual models that employed each variable. The black bars indicate a variable that was also in the generic model. Adjusted time per keystroke and Pause rate were in the generic model but did not figure into any of the individual models.

Other methods for measuring cognitive demand can suffer from limitations such as obtrusiveness, suitability only for infrequent assessment, and a need for special equipment. Our method addresses many of the shortcomings of current assessments and has potential implications for how cognitive demand is measured and monitored. First, this method can unobtrusively gather data by leveraging behaviors in which the individual is already engaged. Next, it is able to capture data continuously over a length of time. This method also requires no special training and uses readily available equipment. Flexibility of the method allows for adjustment to the unique 
characteristics of each individual. The implications of these advantages are manifold. Background data collection means that people can institute a monitoring system without interruption to their daily routine. The unobtrusiveness and continuous nature of the method eases the burden of early, frequent monitoring and therefore facilitates the gathering of baseline data not currently collected. Finally, the realtime monitoring has the potential to allow immediate detection of acute changes, thus enabling the possibility of timely intervention. Just-in-time detection can also help people become more aware of high cognitive demand, training them to eventually cope without need for prompting, thus providing benefits beyond the times they interact with technology.

\subsection{Limitations}

Below we discuss some limitations of this study and mitigation solutions.

As discussed with stress analysis of voice, users might be concerned with the privacy of this method. Although no recording of text is necessary since analysis can be done in real-time, users may perceive a violation of privacy with any type of behavioral monitoring. User education may mitigate this concern along with transparency into what is recorded and how data are used.

The sample in this study was relatively young, possibly affecting external validity. Age can affect reactivity to cognitive demand, but individual characteristics may play an even greater role (Hay and Diehl 2010). Therefore, we believe that the generic model may need to calibrate to age, but our personalized approach would remain the same since it factors in individual differences.

In a study of common stressors, Hay and Diehl (2010) also found that stressor type affected reactivity as well. As we used a stressor inducing one specific type of demand our results might vary with different stressors. Further study of additional high demand tasks is merited.

\section{CONCLUSION}

Other research has examined either keystroke features or linguistic features for high cognitive demand detection, but this research examines this novel combination for classifying cognitive demand status in individuals. The unique combination of features allows a richer understanding of how changes in cognition due to high cognitive demand are expressed in interactions with a computer than either keystroke or linguistic features alone. The results show that the generic model provides a good foundation, but the individualized models were much more powerful because they were tailored to the unique ways in which each individual reacted to high cognitive demand. Although the features that were predictors in the group model and individual models overlapped, there were appreciable differences as well. This variation provides valuable information on interpersonal differences focused on how people are affected by high cognitive demand.

In the future, monitoring of "digital traces" - data passively generated from IT interactions - may be a regular part of a healthy lifestyle (Estrin 2014). This approach provides a low-cost method for cognitive demand monitoring that can be incorporated into everyday activities, and has the potential to assist people in making informed decisions about lifestyle by allowing them to monitor their own behaviors, view trends, and act on that information. Several lines of research follow directly from this current work. Study of additional variables and stressors would lead to better understanding of the processes at work. As people are no longer just using desktop computers, additional studies could include interaction and contextual information about use of mobile devices to build more powerful models. A more 
nuanced understanding of the interactions of activity, environment, stress, workload, and health status can lead to better detection, discrimination, and classification.

The overarching goal of this research is to develop an integrated approach to cognitive demand monitoring using everyday interactions with information technologies. Current cognitive assessment tools and affective computing approaches suffer from drawbacks such as obtrusive monitoring and a need for special equipment, whereas this approach is suited to continuous, unobtrusive monitoring using commonplace technologies. In sum, this research contributes to understanding the interaction between high cognitive demand and text-based technology use, illustrates the high interpersonal variability in that interaction, and has implications for design of cognitive demand monitoring systems for personalized health monitoring and management.

\section{ACKNOWLEDGMENTS}

Drs. A. Ant Ozok, Lina Zhou, Sara Czaja, and Clayton Lewis provided research guidance and manuscript suggestions. Dr. Wanda Pratt's iMed group at the University of Washington, particularly Jordan Eschler, provided further manuscript feedback. Patrick Carrington provided research support. This work was supported in part by a National Science Foundation Graduate Research Fellowship, the National Institutes of Health, National Library of Medicine (NLM) Biomedical and Health Informatics Training Program at the University of Washington (Grant Nr. T15LM007442), and the National Center for Advancing Translational Sciences (NCATS), National Institutes of Health, through Grant Award Number UL1TR001111. The content is solely the responsibility of the authors and does not necessarily represent the official views of the National Science Foundation or National Institutes of Health.

\section{REFERENCES}

Adams, P., Rabbi, M., Rahman, T., Matthews, M., Voida, A., Gay, G., Choudhury, T., Voida, S., 2014. Towards personal stress informatics: Comparing minimally invasive techniques for measuring daily stress in the wild. In Proceedings of Pervasive Computing Technologies for Healthcare.

American Psychological Association. 2015. Stress in America: Paying with our health.

Baddeley, A., 2003. Working memory and language: an overview. J. Commun. Disord. 36, 3 (May 2003), 189-208.

Bird, S., Klein, E., Loper, E., 2009. Natural language processing with Python, O'Reilly.

Bourne, L., Yaroush R., 2003. Stress and cognition: A cognitive psychological perspective. Unpubl. Manuscr. NASA Grant NAG2-1561 (2003).

Byers, J., Bittner, A., Hill, S., 1989. Traditional and raw task load index (TLX) correlations: Are paired comparisons necessary? In Advances in industrial ergonomics \& safety. London: Taylor \& Francis, 481-485.

Card, S., Moran, T., Newell, A., 1986. The model human processor: An engineering model of human performance. Handb. Hum. Percept. 2 (1986).

Carneiro, D., Castillo, J., Novais, P., Fernández-Caballero, A., Neves, J., 2012. Multimodal behavioral analysis for non-invasive stress detection. Expert Syst. Appl. 39, 18 (2012), 13376-13389.

Cohen, S., Kessler, R., Gordon, L., 1995. Strategies for measuring stress in studies of psychiatric and physical disorders. Meas. Stress Guide Health Soc. Sci. (1995), 3-26.

DePaulo, B., Lindsay, J., Malone, B., Muhlenbruck, L., Charlton, K., Cooper, H., 2003. Cues to deception. Psychol. Bull. 129, 1 (2003).

Dinges, D., Venkataraman, S., McGlinchey, E., Metaxas, D., 2007. Monitoring of facial stress during space flight: Optical computer recognition combining discriminative and generative methods. Acta Astronaut. 60, 4 (2007), 341-350.

Dobbs, A., Rule, B., 1989. Adult age differences in working memory. Psychol. Aging 4, 4 (1989), 500.

Epp, C., Lippold, M., Mandryk, R., 2011. Identifying emotional states using keystroke dynamics. In Proceedings of the SIGCHI Conference on Human Factors in Computing Systems. 715-724.

Estrin, D. 2014. Small Date, Where N=Me. Communications of the ACM. 57, 4 (2014), 32-34.

Eysenck, M., Derakshan, N., Santos, R., Calvo, M., 2007. Anxiety and cognitive performance: attentional control theory. Emotion 7, 2 (2007), 336.

Feild, H., Allan. J., Jones, R. 2010. Predicting Searcher Frustration. In Proceedings of the ACM SIGIR Conference on Research and Development in Information Retrieval. 34-41.

Fox, S., Duggan, M., 2013. Tracking for Health. Pew Res. Cent. Internet Am. Life Proj. (January 2013).

Gawron, V., 2000. Human performance measures handbook, Psychology Press.

Goh, J., Pfeffer, J., Zenios, S., 2015. The Relationship Between Workplace Stressors and Mortality and Health Costs in the United States. Manag. Sci. (March 2015).

Goldberger, L., Breznitz, S., 2010. Handbook of stress, Simon and Schuster. 
Gunetti, D., Picardi, C., 2005. Keystroke analysis of free text. ACM Trans. Inf. Syst. Secur. TISSEC 8, 3 (2005), 312-347.

Hancock, P., 1989. A dynamic model of stress and sustained attention. Hum. Factors J. Hum. Factors Ergon. Soc. 31, 5 (1989), 519-537.

Hart, S., Staveland, L., 1988. Development of NASA-TLX (Task Load Index): Results of empirical and theoretical research. Hum. Ment. Workload 1, 3 (1988), 139-183.

Hay, E., Diehl, M., 2010. Reactivity to Daily Stressors in Adulthood: The Importance of Stressor Type in Characterizing Risk Factors. Psychol. Aging. 25, 1 (2010), 118-131.

Healey, J., Picard, R., 2000. Smartcar: detecting driver stress. In Pattern Recognition, 2000. Proceedings 15th International Conference on. 218-221.

Hernandez, J., Paredes, P., Roseway, A., Czerwinski, M. 2014. Under Pressure, Sensing Stress of Computer Users. In Proceedings of the SIGCHI Conference on Human Factors in Computing Systems. 51-60.

Hone, K., Graham, R., 2000. Towards a Tool for the Subjective Assessment of Speech System Interfaces (SASSI). Nat Lang Eng 6, 3-4 (September 2000), 287-303.

Hosmer Jr, D., Lemeshow, S., Sturdivant, R., 2013. Applied logistic regression, Wiley. com.

Jain, A., Duin, R., Mao, J., 2000. Statistical pattern recognition: A review. Pattern Anal. Mach. Intell. IEEE Trans. On 22, 1 (2000), 4-37.

Karasek, R., Brisson, C., Kawakami, N., Houtman, I., Bongers, P., Amick, B., 1998. The Job Content Questionnaire (JCQ): an instrument for internationally comparative assessments of psychosocial job characteristics. J. Occup. Health Psychol. 3, 4 (1998), 322.

Khawaja, M., Chen, F., 2010. Using language complexity to measure cognitive load for adaptive interaction design. (2010), 333-336.

Khawaja, M., Chen, F., Marcus, N., 2013. Measuring Cognitive Load Using Linguistic Features: Implications for Usability Evaluation and Adaptive Interaction Design. Int. J. Hum.-Comput. Interact. 30, 5 (November 2013), 343-368.

Kolakowska, A. 2013. A review of emotion recognition methods based on keystroke dynamics and mouse movements. In Proceedings of the IEEE International Conference on Human System Interaction. 548-555.

Lu, H., Frauendorfer, D., Rabbi, M., Mast, M., Chittaranjan, G., Campbell, A., Gatica-Perez, D., Choudhury, T., 2012. StressSense: Detecting stress in unconstrained acoustic environments using smartphones. In 351-360.

Lupien, S., Maheu, F., Tu, M., Fiocco, A., Schramek, T., 2007. The effects of stress and stress hormones on human cognition: implications for the field of brain and cognition. Brain Cogn. 65, 3 (2007), 209237.

Mandler, G., 2010. Thought, memory, and learning: Effects of emotional stress. In The Handbook of Stress. New York, NY: The Free Press.

Mark, G., Gudith, D., Klocke, U., 2008. The Cost of Interrupted Work: More Speed and Stress. In ACM, 107-110.

Mark, G., Voida, S., Cardello, A., 2012. "A pace not dictated by electrons": an empirical study of work without email. In 555-564.

Matthews, G., Campbell, S., Falconer, S., Joyner, L., Huggins, J., Gilliland, K., Grier, R., Warm, J., 2002. Fundamental dimensions of subjective state in performance settings: task engagement, distress, and worry. Emotion 2, 4 (2002), 315.

Mehler, B., Reimer, B., Coughlin, J., 2012. Sensitivity of Physiological Measures for Detecting Systematic Variations in Cognitive Demand From a Working Memory Task An On-Road Study Across Three Age Groups. Hum. Factors J. Hum. Factors Ergon. Soc. 54, 3 (June 2012), 396-412.

Mehrabian, A., Wiener, M., 1966. Non-immediacy between communicator and object of communication in a verbal message: Application to the inference of attitudes. J. Consult. Psychol. 30, 5 (1966), 420.

Menard, S., 2002. Applied logistic regression analysis, Sage.

Monrose, F., Rubin, A., 1997. Authentication via keystroke dynamics. In Proceedings of the 4th ACM conference on Computer and communications security. 48-56.

Monrose, F., Rubin, A., 2000. Keystroke dynamics as a biometric for authentication. Future Gener. Comput. Syst. 16, 4 (2000), 351-359.

Moroney, W., Biers, D., Eggemeier, F., Mitchell, J., 1992. A comparison of two scoring procedures with the NASA task load index in a simulated flight task. In Aerospace and Electronics Conference, 1992. NAECON 1992. 734-740.

Oviatt, S., 2006. Human-centered design meets cognitive load theory: designing interfaces that help people think. In ACM, 871-880.

Owen, A., McMillan, K., Laird, A., Bullmore, E., 2005. N-back working memory paradigm: A meta-analysis of normative functional neuroimaging studies. Hum. Brain Mapp. 25, 1 (2005), 46-59.

Pennebaker, J., Francis, M., Booth, R., 2001. Linguistic inquiry and word count: LIWC 2001. Mahway Lawrence Erlbaum Assoc. (2001), 71.

Pew Research Internet Project, 2014. Device Ownership Over Time. (2014). Retrieved November 11, 2014 from http://www.pewinternet.org/data-trend/mobile/device-ownership/ 
Qi, Y., Reynolds, C., Picard, R., 2001. The Bayes Point Machine for computer-user frustration detection via pressuremouse. In Proceedings of the 2001 workshop on Perceptive user interfaces. 1-5.

Schilperoord, J., 2002. On the Cognitive Status of Pauses in Discourse Production. T. Olive \& C. Levy, eds. Contemporary Tools and Techniques for Studying Writing. Studies in Writing. Springer Netherlands, 61-87.

Semmer, N., McGrath, J., Beehr, T., 2005. Conceptual Issues in Research on Stress and Health. In Handbook of Stress Medicine and Health.

Smith, A. 1982. Symbol Digit Modalities Test. Western Psychological Services, Los Angeles.

Smith, A., 2015. U.S. Smartphone Use in 2015, Pew Research Center.

Sweller, J., 1988. Cognitive load during problem solving: Effects on learning. Cogn. Sci. 12 (1988), 257285.

Sweller, J., Ayres, P., Kalyuga, S., 2011. Cognitive Load Theory, New York, NY: Springer New York.

Varvogli, L., Darviri, C., 2011. Stress Management Techniques: evidence-based procedures that reduce stress and promote health. Health Sci. J. 5, 2 (2011), 74-89.

M. Villani, C. Tappert, G. Ngo, J. Simone, H. St Fort, and S. Cha. 2006. Keystroke biometric recognition studies on long-text input under ideal and application-oriented conditions. In Computer Vision and Pattern Recognition Workshop, 2006. CVPRW'06. 39-39.

Vizer, L., Zhou, L., Sears, A., 2009. Automated stress detection using keystroke and linguistic features: An exploratory study. Int. J. Hum.-Comput. Stud. 67, 10 (2009), 870-886.

Vizer, L., 2013. Different Strokes for Different Folks: Individual Stress Response As Manifested in Typed Text. In CHI '13 Extended Abstracts on Human Factors in Computing Systems. CHI EA '13. New York, NY, USA: ACM, 2773-2778.

Vizer, L., Sears, A., 2015. Automatic Classification of Spontaneous Text-Based Computer Interactions From Older Adults With and Without Pre-Mild Cognitive Impairment. IEEE Pervasive Comput. $14: 4,64-71$.

Williams, S., Cooper, C., 1998. Measuring occupational stress: development of the pressure management indicator. J. Occup. Health Psychol. 3, 4 (1998), 306.

Wilson, G., 2002. Psychophysiological test methods and procedures. In TG O'Brien, ed. Handbook of human factors testing and evaluation. Mahwah, NJ: Lawrence Erlbaum, 127-156.

Witten, I., Frank, E., Hall, M., 2011. Data Mining: Practical Machine Learning Tools and Techniques: Practical Machine Learning Tools and Techniques, Elsevier.

Yin, B., Ruiz, N., Chen, F., Khawaja, M., 2007. Automatic cognitive load detection from speech features. In Proceedings of the 19th Australasian conference on Computer-Human Interaction: Entertaining User Interfaces. 249-255.

Zhou, L., 2005. An Empirical Investigation of Deception Behavior in Instant Messaging. IEEE Trans. Prof. Commun. 48, 2 (June 2005), 147-160.

Zhou, L., Burgoon, J., Nunamaker, J., Twitchell, D., 2004. Automating Linguistics-Based Cues for Detecting Deception in Text-Based Asynchronous Computer-Mediated Communications. Group Decis. Negot. 13, 1 (January 2004), 81-106.

Zhou, L., Burgoon, J., Twitchell, D., Qin, T., Nunamaker Jr, J., 2004. A comparison of classification methods for predicting deception in computer-mediated communication. J. Manag. Inf. Syst. 20, 4 (2004), 139-166.

Zhou, L., Twitchell, D., Qin, T., Burgoon, J., and Nunamaker Jr, J., 2003. An exploratory study into deception detection in text-based computer-mediated communication. In System Sciences, 2003. Proceedings of the 36th Annual Hawaii International Conference on System Sciences. 10-pp.

Zhou, L., Zhang, D., 2006. A comparison of deception behavior in dyad and triadic group decision making in synchronous computer-mediated communication. Small Group Res. 37, 2 (2006), 140-164.

Zhou, L., Zhang, D., 2008. Following linguistic footprints: automatic deception detection in online communication. Commun. ACM 51, 9 (September 2008), 119-122.

Zuckerman, M., DePaulo, B., Rosenthal, R., 1981. Verbal and nonverbal communication of deception. Adv. Exp. Soc. Psychol. 14, 1 (1981), 59.

Zuckerman, M., Driver, R., 1985. Telling Lies: Verbal and nonverbal correlates of deception. In Nonverbal Communication: An Integrated Perspective. Hillsdale, NJ: Lawrence Erlbaum.

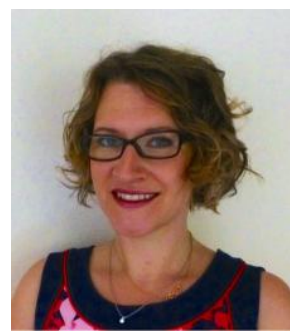

Lisa M. Vizer is a Research Assistant Professor in the Division of Internal Medicine at UNC-Chapel Hill. She earned both a BS degree in Computer Science and a MHCI degree from Carnegie Mellon. After spending several years working in industry as a usability professional, she returned to school to pursue a PhD in Information Systems, granted in 2013. Her work focuses on the patient-centered ecology of using passively collected behavior data to support patients with brain disorders and trauma outside the clinic. Dr. Vizer is a member of AMIA, ACM, Phi Beta Kappa, 
and Phi Kappa Phi.

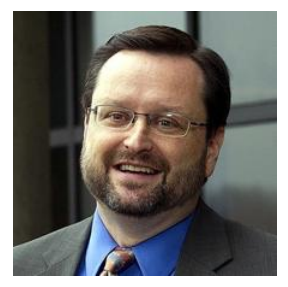

Andrew Sears is Dean of the College of Information Sciences and Technology at Pennsylvania State University. His research interests include issues related to human-centered computing and accessibility, mobile computing, health information technologies, speech recognition, managing interruptions while working with information technologies, and assessing an individual's cognitive status via normal daily interactions with information technologies. Sears has a PhD in computer science from the University of Maryland, College Park. He's a member of the ACM. 


\section{APPENDIX A}

\section{Means and Variability for Generic Cognitive Demand Protocol}

\begin{tabular}{|c|c|c|c|c|c|c|}
\hline \multirow[b]{2}{*}{ Variables } & \multicolumn{2}{|c|}{ Control $(\mathrm{N}=80)$} & \multicolumn{2}{|c|}{$\begin{array}{c}\text { Experimental } \\
\quad(\mathrm{N}=80)\end{array}$} & \multicolumn{2}{|c|}{ T-test } \\
\hline & Mean & (SD) & Mean & (SD) & $\mathbf{T}$ & $p$ \\
\hline Total duration & -0.29 & $(1.34)$ & -0.023 & $(2.03)$ & -0.975 & 0.331 \\
\hline Pause rate & -0.29 & $(1.10)$ & -0.23 & $(1.01)$ & -0.311 & 0.756 \\
\hline Time per key & -0.39 & $(1.39)$ & -0.036 & $(2.57)$ & -1.071 & 0.286 \\
\hline Adjusted time per key & -0.29 & $(1.12)$ & 0.17 & $(2.95)$ & -1.343 & 0.183 \\
\hline Time per pause & -0.29 & $(1.12)$ & 0.17 & $(2.95)$ & -1.283 & 0.201 \\
\hline Word count & 0.48 & $(2.44)$ & 0.23 & $(1.12)$ & 0.856 & 0.393 \\
\hline Words per sentence & 0.39 & $(1.26)$ & 0.088 & $(1.37)$ & 1.470 & 0.143 \\
\hline Words over 6 letters & 0.042 & $(0.98)$ & -0.12 & $(1.14)$ & 0.992 & 0.323 \\
\hline Correction key rate & -0.15 & $(1.23)$ & -0.11 & $(1.35)$ & -0.234 & 0.815 \\
\hline Function key rate & 0.032 & $(0.99)$ & -0.022 & $(1.20)$ & 0.310 & 0.757 \\
\hline Other key rate & 0.069 & $(1.16)$ & 0.049 & $(1.45)$ & 0.093 & 0.926 \\
\hline Lexical diversity & 0.030 & $(1.60)$ & -0.033 & $(1.01)$ & 0.298 & 0.766 \\
\hline Cognitive processes & 0.22 & $(1.06)$ & 0.35 & $(1.19)$ & -0.721 & 0.472 \\
\hline Insight & 0.16 & $(1.01)$ & 0.24 & $(1.02)$ & -0.449 & 0.654 \\
\hline Certainty & 0.046 & $(1.05)$ & -0.19 & $(1.28)$ & 1.278 & 0.203 \\
\hline Tentativeness & 0.18 & $(1.11)$ & 0.048 & $(1.10)$ & 0.778 & 0.438 \\
\hline Perception & -0.24 & $(1.26)$ & -0.31 & $(0.82)$ & 0.404 & 0.686 \\
\hline Positive emotion & 0.022 & $(1.07)$ & -0.013 & $(1.24)$ & -0.187 & 0.852 \\
\hline Negative emotion & 0.33 & $(1.63)$ & 0.97 & $(2.39)$ & -1.977 & 0.050 \\
\hline First-person pronouns & 0.144 & $(1.46)$ & 0.269 & $(1.95)$ & 0.459 & 0.647 \\
\hline Third-person pronouns & 0.026 & $(1.39)$ & 0.018 & $(2.25)$ & 0.028 & 0.977 \\
\hline
\end{tabular}

Table A: Feature statistics for cognitive demand (* $p<0.0025)$. Highlighted variables were selected for the logistic regression model. 
APPENDIX B

Means and Variability for Cognitive Demand - Extended Protocol

\begin{tabular}{|c|c|c|c|c|c|c|}
\hline \multirow{2}{*}{$\begin{array}{l}\text { Participant } 1 \\
\text { Variables }\end{array}$} & \multicolumn{2}{|c|}{ Control $(\mathrm{N}=30)$} & \multicolumn{2}{|c|}{$\begin{array}{l}\text { Experimental } \\
\quad(\mathrm{N}=28)\end{array}$} & \multicolumn{2}{|c|}{ T-test } \\
\hline & Mean & (SD) & Mean & (SD) & $\mathbf{T}$ & $p$ \\
\hline Total duration & 146.79 & $(5.88)$ & 148.91 & $(19.54)$ & -0.599 & 0.554 \\
\hline Pause rate & 0.013 & $(0.0047)$ & 0.015 & $(0.0047)$ & -1.578 & 0.126 \\
\hline Time per pause & 0.89 & $(0.32)$ & 1.21 & $(1.58)$ & -1.098 & 0.282 \\
\hline Time per key & 0.16 & $(0.0061)$ & 0.17 & $(0.017)$ & -1.039 & 0.308 \\
\hline Adjusted time per key & 0.16 & $(0.0056)$ & 0.17 & $(0.011)$ & -1.443 & 0.160 \\
\hline Word count & 156.41 & $(6.02)$ & 158.45 & $(30.72)$ & -0.333 & 0.742 \\
\hline Words per sentence & 23.18 & $(5.84)$ & 29.17 & (13.09) & -2.122 & 0.043 \\
\hline Words over 6 letters & 11.19 & $(3.07)$ & 12.65 & $(2.04)$ & -2.333 & 0.027 \\
\hline Correction key rate & 0.064 & $(0.014)$ & 0.062 & $(0.014)$ & 0.371 & 0.714 \\
\hline Function key rate & 0.0071 & $(0.0085)$ & 0.0050 & $(0.0099)$ & 1.207 & 0.238 \\
\hline Other key rate & 0.93 & $(0.014)$ & 0.93 & $(0.018)$ & -0.937 & 0.357 \\
\hline Cognitive processes & 21.72 & $(4.23)$ & 23.85 & $(3.57)$ & -2.995 & 0.006 \\
\hline Insight & 3.18 & $(1.64)$ & 3.38 & (1.49) & -0.502 & 0.619 \\
\hline Certainty & 1.55 & $(1.11)$ & 1.54 & $(0.87)$ & 0.034 & 0.973 \\
\hline Tentativeness & 3.56 & $(1.23)$ & 3.67 & (1.88) & -0.252 & 0.803 \\
\hline Lexical diversity & 24.76 & (1.88) & 27.38 & $(4.83)$ & -2.767 & 0.008 \\
\hline Perception & 1.64 & (1.14) & 1.62 & $(1.12)$ & 0.045 & 0.965 \\
\hline Positive emotion & 3.57 & $(1.62)$ & 3.28 & $(1.85)$ & 0.722 & 0.477 \\
\hline Negative emotion & 1.46 & $(1.11)$ & 2.61 & $(1.55)$ & -3.815 & $0.001 *$ \\
\hline First-person pronouns & 5.47 & $(5.41)$ & 5.32 & $(5.49)$ & 0.110 & 0.913 \\
\hline Third-person pronouns & 0.43 & $(0.85)$ & 0.41 & $(0.88)$ & 0.108 & 0.915 \\
\hline
\end{tabular}

Table B-1: Feature statistics for Participant $1(* p<0.0025)$. Highlighted variables were selected for the logistic regression model. 


\begin{tabular}{|c|c|c|c|c|c|c|}
\hline \multirow{2}{*}{$\begin{array}{l}\text { Participant } 2 \\
\text { Variables }\end{array}$} & \multicolumn{2}{|c|}{ Control $(\mathrm{N}=30)$} & \multicolumn{2}{|c|}{$\begin{array}{c}\text { Experimental } \\
\quad(\mathrm{N}=30)\end{array}$} & \multicolumn{2}{|c|}{ T-test } \\
\hline & Mean & (SD) & Mean & (SD) & $\mathbf{T}$ & $p$ \\
\hline Total duration & 284.05 & $(51.83)$ & 333.71 & $(58.58)$ & -4.732 & 0.000 * \\
\hline Pause rate & 0.076 & $(0.023)$ & 0.081 & $(0.021)$ & -0.950 & 0.350 \\
\hline Time per pause & 0.95 & $(0.26)$ & 1.16 & $(0.28)$ & -3.903 & 0.001 * \\
\hline Time per key & 0.27 & $(0.019)$ & 0.28 & $(0.037)$ & -1.680 & 0.104 \\
\hline Adjusted time per key & 0.21 & $(0.0066)$ & 0.22 & $(0.014)$ & -1.407 & 0.170 \\
\hline Word count & 155.53 & (25.38) & 180.37 & $(27.92)$ & -4.460 & 0.000 * \\
\hline Words per sentence & 18.47 & $(3.89)$ & 17.59 & $(3.14)$ & 1.067 & 0.295 \\
\hline Words over 6 letters & 17.32 & $(4.18)$ & 17.29 & $(3.51)$ & 0.027 & 0.979 \\
\hline Correction key rate & 0.033 & $(0.0062)$ & 0.033 & $(0.0071)$ & 0.271 & 0.788 \\
\hline Function key rate & 0.076 & $(0.023)$ & 0.081 & $(0.021)$ & 0.125 & 0.901 \\
\hline Other key rate & 0.082 & $(0.022)$ & 0.081 & $(0.020)$ & -0.290 & 0.774 \\
\hline Lexical diversity & 20.15 & $(2.55)$ & 23.16 & $(3.12)$ & -4.099 & 0.000 * \\
\hline Cognitive processes & 15.78 & $(3.88)$ & 17.13 & $(3.60)$ & -1.309 & 0.201 \\
\hline Insight & 2.40 & $(1.47)$ & 2.92 & $(1.50)$ & -1.259 & 0.218 \\
\hline Certainty & 2.12 & $(1.31)$ & 2.55 & $(1.20)$ & -1.441 & 0.160 \\
\hline Tentativeness & 2.53 & (1.43) & 2.86 & (1.35) & -0.814 & 0.423 \\
\hline Perception & 2.05 & $(1.66)$ & 2.23 & $(1.37)$ & -0.441 & 0.663 \\
\hline Positive emotion & 4.05 & $(2.03)$ & 3.99 & $(1.90)$ & 0.115 & 0.909 \\
\hline Negative emotion & 2.08 & $(1.64)$ & 1.44 & (1.33) & 1.591 & 0.123 \\
\hline First-person pronouns & 2.86 & $(3.26)$ & 3.49 & $(3.49)$ & 0.730 & 0.468 \\
\hline Third-person pronouns & 1.34 & $(1.78)$ & 1.12 & (1.09) & 0.575 & 0.569 \\
\hline
\end{tabular}

Table B-2: Feature statistics for Participant 2 (*p<0.0025). Highlighted variables were selected for the logistic regression model. 


\begin{tabular}{|c|c|c|c|c|c|c|}
\hline \multirow{2}{*}{$\begin{array}{l}\text { Participant } 3 \\
\text { Variables }\end{array}$} & \multicolumn{2}{|c|}{ Control $(\mathrm{N}=30)$} & \multicolumn{2}{|c|}{$\begin{array}{c}\text { Experimental } \\
(N=30)\end{array}$} & \multicolumn{2}{|c|}{ T-test } \\
\hline & Mean & (SD) & Mean & (SD) & $\mathbf{T}$ & $p$ \\
\hline Total duration & 484.69 & $(92.96)$ & 454.25 & (104.16) & 1.215 & 0.234 \\
\hline Pause rate & 0.16 & $(0.036)$ & 0.15 & $(0.036)$ & 0.611 & 0.546 \\
\hline Time per pause & 0.23 & $(0.053)$ & 0.22 & $(0.059)$ & 0.674 & 0.506 \\
\hline Time per key & 0.39 & $(0.029)$ & 0.37 & $(0.048)$ & 1.836 & 0.077 \\
\hline Adjusted time per key & 0.26 & $(0.0098)$ & 0.26 & $(0.025)$ & 1.529 & 0.137 \\
\hline Lexical diversity & 19.95 & (1.96) & 20.66 & $(2.47)$ & -1.235 & 0.222 \\
\hline Word count & 137.80 & $(17.31)$ & 138.07 & $(16.72)$ & -0.060 & 0.952 \\
\hline Words per sentence & 18.44 & $(4.09)$ & 20.03 & $(5.45)$ & -1.177 & 0.249 \\
\hline Words over 6 letters & 16.45 & $(4.84)$ & 14.78 & $(3.60)$ & 1.644 & 0.111 \\
\hline Positive emotion & 3.67 & $(2.19)$ & 3.68 & $(2.20)$ & -0.003 & 0.998 \\
\hline Negative emotion & 0.96 & (1.04) & 1.90 & $(2.64)$ & -1.767 & 0.088 \\
\hline Cognitive processes & 17.82 & $(4.46)$ & 19.58 & $(4.21)$ & -1.626 & 0.115 \\
\hline Insight & 3.24 & $(2.46)$ & 3.83 & $(1.72)$ & -1.408 & 0.170 \\
\hline Tentativeness & 3.53 & $(2.06)$ & 4.16 & $(1.82)$ & -1.170 & 0.251 \\
\hline Certainty & 0.84 & $(0.87)$ & 0.97 & $(0.71)$ & -0.612 & 0.545 \\
\hline Perception & 2.54 & $(3.31)$ & 1.78 & $(1.39)$ & 1.209 & 0.236 \\
\hline Function key rate & 0.043 & $(0.0097)$ & 0.039 & $(0.012)$ & 1.329 & 0.194 \\
\hline Correction key rate & 0.21 & $(0.046)$ & 0.21 & $(0.066)$ & 0.276 & 0.785 \\
\hline Other key rate & 0.74 & $(0.044)$ & 0.75 & $(0.067)$ & -0.570 & 0.573 \\
\hline First-person pronouns & 4.94 & $(5.33)$ & 5.28 & $(5.18)$ & 0.253 & 0.802 \\
\hline Third-person pronouns & 1.94 & $(2.30)$ & 1.61 & $(1.61)$ & 0.440 & 0.662 \\
\hline
\end{tabular}

Table B-3: Feature statistics for Participant $3(* p<0.0025)$. Highlighted variables were selected for the logistic regression model. 


\begin{tabular}{|c|c|c|c|c|c|c|}
\hline \multirow{2}{*}{$\begin{array}{l}\text { Participant } 4 \\
\text { Variables }\end{array}$} & \multicolumn{2}{|c|}{ Control (N=30) } & \multicolumn{2}{|c|}{$\begin{array}{c}\text { Experimental } \\
(\mathrm{N}=30)\end{array}$} & \multicolumn{2}{|c|}{ T-test } \\
\hline & Mean & (SD) & Mean & (SD) & $\mathbf{T}$ & $p$ \\
\hline Total duration & 154.57 & $(9.71)$ & 164.99 & (11.83) & -0.191 & 0.850 \\
\hline Pause rate & 0.013 & $(0.0053)$ & 0.013 & $(0.0055)$ & -0.144 & 0.887 \\
\hline Time per pause & 0.18 & $(0.010)$ & 0.18 & $(0.0095)$ & 0.046 & 0.964 \\
\hline Time per key & 0.19 & $(0.0097)$ & 0.19 & $(0.0081)$ & -0.072 & 0.943 \\
\hline Adjusted time per key & 0.19 & $(0.0093)$ & 0.19 & $(0.0071)$ & 0.185 & 0.855 \\
\hline Word count & 146.63 & $(8.41)$ & 147.43 & $(10.54)$ & -0.300 & 0.766 \\
\hline Words per sentence & 13.72 & $(3.81)$ & 11.00 & $(2.52)$ & 3.230 & 0.003 \\
\hline Words over 6 letters & 13.48 & $(3.00)$ & 10.57 & (2.99) & 3.780 & 0.001 * \\
\hline Correction key rate & 0.031 & $(0.0091)$ & 0.038 & $(0.011)$ & -2.895 & 0.007 \\
\hline Function key rate & 0.040 & $(0.0098)$ & 0.040 & $(0.0098)$ & -0.150 & 0.881 \\
\hline Other key rate & 0.93 & $(0.013)$ & 0.92 & $(0.012)$ & 2.341 & 0.026 \\
\hline Lexical diversity & 17.90 & $(2.30)$ & 18.45 & $(2.08)$ & -0.980 & 0.331 \\
\hline Cognitive processes & 17.67 & $(4.29)$ & 19.49 & $(3.76)$ & -2.039 & 0.051 \\
\hline Insight & 2.82 & $(1.54)$ & 3.74 & $(1.71)$ & -2.675 & 0.012 \\
\hline Certainty & 0.95 & $(0.95)$ & 0.90 & $(0.70)$ & 0.257 & 0.799 \\
\hline Tentativeness & 3.99 & $(1.39)$ & 5.35 & $(2.46)$ & -2.401 & 0.023 \\
\hline Perception & 2.06 & $(1.61)$ & 1.75 & $(1.51)$ & 0.855 & 0.400 \\
\hline Positive emotion & 4.20 & $(2.03)$ & 5.23 & $(2.72)$ & -1.585 & 0.124 \\
\hline Negative emotion & 1.20 & $(1.05)$ & 2.27 & $(1.38)$ & -3.323 & 0.002 * \\
\hline First-person pronouns & 5.50 & $(5.07)$ & 6.06 & $(5.99)$ & 0.394 & 0.695 \\
\hline Third-person pronouns & 2.23 & $(2.90)$ & 1.02 & $(1.23)$ & 2.384 & 0.024 \\
\hline
\end{tabular}

Table B-4: Feature statistics for Participant $4\left({ }^{*} p<0.0025\right)$. Highlighted variables were selected for the logistic regression model. 


\begin{tabular}{|c|c|c|c|c|c|c|}
\hline \multirow{2}{*}{$\begin{array}{l}\text { Participant } 5 \\
\text { Variables }\end{array}$} & \multicolumn{2}{|c|}{ Control (N=30) } & \multicolumn{2}{|c|}{$\begin{array}{c}\text { Experimental } \\
(\mathrm{N}=30)\end{array}$} & \multicolumn{2}{|c|}{ T-test } \\
\hline & Mean & (SD) & Mean & (SD) & $\mathbf{T}$ & $p$ \\
\hline Total duration & 159.40 & (10.38) & 174.06 & $(66.72)$ & -1.234 & 0.227 \\
\hline Pause rate & 0.028 & $(0.0069)$ & 0.026 & $(0.0075)$ & 0.856 & 0.399 \\
\hline Time per pause & 0.21 & $(0.056)$ & 0.20 & $(0.057)$ & -0.538 & 0.595 \\
\hline Time per key & 0.19 & $(0.011)$ & 0.20 & $(0.062)$ & -0.833 & 0.412 \\
\hline Adjusted time per key & 0.18 & $(0.0078)$ & 0.18 & $(0.017)$ & -0.728 & 0.473 \\
\hline Word count & 151.07 & $(10.25)$ & 152.33 & $(7.36)$ & -0.596 & 0.556 \\
\hline Words per sentence & 17.10 & $(3.48)$ & 16.89 & $(3.50)$ & 0.292 & 0.772 \\
\hline Words over 6 letters & 12.30 & $(3.76)$ & 13.10 & $(3.17)$ & -0.969 & 0.341 \\
\hline Correction key rate & 0.033 & $(0.010)$ & 0.044 & $(0.023)$ & -2.442 & 0.021 \\
\hline Function key rate & 0.028 & $(0.0069)$ & 0.026 & $(0.0075)$ & 0.967 & 0.342 \\
\hline Other key rate & 0.94 & $(0.011)$ & 0.93 & $(0.021)$ & 2.233 & 0.033 \\
\hline Lexical diversity & 21.45 & $(1.64)$ & 21.31 & $(1.64)$ & 0.331 & 0.742 \\
\hline Cognitive processes & 19.54 & $(2.58)$ & 16.76 & $(4.00)$ & 3.395 & $0.002 *$ \\
\hline Insight & 2.64 & $(1.76)$ & 2.13 & $(1.27)$ & 1.350 & 0.188 \\
\hline Certainty & 1.12 & $(0.94)$ & 0.70 & $(0.77)$ & 1.751 & 0.091 \\
\hline Tentativeness & 3.27 & $(1.46)$ & 2.53 & $(1.35)$ & 1.895 & 0.068 \\
\hline Perception & 1.33 & $(1.22)$ & 1.96 & (1.65) & -1.555 & 0.131 \\
\hline Positive emotion & 3.72 & $(2.21)$ & 3.43 & $(1.51)$ & 0.681 & 0.501 \\
\hline Negative emotion & 2.08 & $(1.78)$ & 2.17 & $(1.39)$ & -0.206 & 0.838 \\
\hline First-person pronouns & 5.68 & $(6.16)$ & 5.69 & $(5.80)$ & 0.0065 & 0.995 \\
\hline Third-person pronouns & 1.56 & $(2.33)$ & 1.20 & $(2.63)$ & 0.598 & 0.554 \\
\hline
\end{tabular}

Table B-5: Feature statistics for Participant $5\left({ }^{*} p<0.0025\right)$. Highlighted variables were selected for the logistic regression model. 


\begin{tabular}{|c|c|c|c|c|c|c|}
\hline \multirow{2}{*}{$\begin{array}{l}\text { Participant } 6 \\
\text { Variables }\end{array}$} & \multicolumn{2}{|c|}{ Control $(\mathrm{N}=30)$} & \multicolumn{2}{|c|}{$\begin{array}{c}\text { Experimental } \\
(\mathrm{N}=30)\end{array}$} & \multicolumn{2}{|c|}{ T-test } \\
\hline & Mean & (SD) & Mean & (SD) & $\mathbf{T}$ & $p$ \\
\hline Total duration & 296.29 & $(34.65)$ & 308.78 & $(59.17)$ & -0.891 & 0.380 \\
\hline Pause rate & 0.093 & $(0.015)$ & 0.098 & $(0.024)$ & -0.868 & 0.393 \\
\hline Time per pause & 1.27 & $(0.30)$ & 1.38 & $(0.49)$ & -0.991 & 0.330 \\
\hline Time per key & 0.33 & $(0.031)$ & 0.34 & $(0.060)$ & -0.697 & 0.491 \\
\hline Adjusted time per key & 0.23 & $(0.010)$ & 0.23 & $(0.017)$ & -1.182 & 0.247 \\
\hline Word count & 146.93 & $(9.78)$ & 151.13 & (11.32) & -1.603 & 0.120 \\
\hline Words per sentence & 86.44 & $(51.27)$ & 81.48 & $(55.20)$ & 0.575 & 0.570 \\
\hline Words over 6 letters & 14.18 & $(3.57)$ & 12.44 & $(4.35)$ & 2.047 & 0.050 \\
\hline Correction key rate & 0.070 & $(0.018)$ & 0.078 & $(0.021)$ & -1.663 & 0.107 \\
\hline Function key rate & 0.011 & $(0.0046)$ & 0.011 & $(0.0038)$ & 1.013 & 0.320 \\
\hline Other key rate & 0.92 & $(0.020)$ & 0.91 & $(0.021)$ & 1.3387 & 0.176 \\
\hline Lexical diversity & 22.69 & $(2.61)$ & 23.07 & (1.94) & -0.642 & 0.524 \\
\hline Cognitive processes & 14.22 & $(3.00)$ & 15.44 & $(4.11)$ & -1.459 & 0.155 \\
\hline Insight & 1.51 & $(1.37)$ & 1.81 & $(1.43)$ & -0.964 & 0.343 \\
\hline Certainty & 0.95 & $(1.01)$ & 1.15 & $(0.96)$ & -0.829 & 0.414 \\
\hline Tentativeness & 1.55 & $(0.97)$ & 1.34 & $(1.29)$ & 0.715 & 0.480 \\
\hline Perception & 1.83 & $(2.18)$ & 1.60 & $(1.34)$ & 0.471 & 0.641 \\
\hline Positive emotion & 2.48 & $(1.82)$ & 2.12 & $(1.37)$ & 1.254 & 0.220 \\
\hline Negative emotion & 1.32 & $(0.88)$ & 1.88 & (1.13) & -2.458 & 0.020 \\
\hline First-person pronouns & 9.41 & $(3.56)$ & 9.78 & $(4.00)$ & 0.383 & 0.704 \\
\hline Third-person pronouns & 2.51 & $(1.75)$ & 3.70 & $(3.33)$ & -1.678 & 0.104 \\
\hline
\end{tabular}

Table B-6: Feature statistics for Participant $6(* p<0.0025)$. Highlighted variables were selected for the logistic regression model. 


\begin{tabular}{|c|c|c|c|c|c|c|}
\hline \multirow{2}{*}{$\begin{array}{l}\text { Participant } 7 \\
\text { Variables }\end{array}$} & \multicolumn{2}{|c|}{ Control $(\mathrm{N}=30)$} & \multicolumn{2}{|c|}{$\begin{array}{c}\text { Experimental } \\
(\mathrm{N}=30)\end{array}$} & \multicolumn{2}{|c|}{ T-test } \\
\hline & Mean & (SD) & Mean & (SD) & $\mathbf{T}$ & $p$ \\
\hline Total duration & 204.79 & $(27.87)$ & 206.39 & $(55.92)$ & -0.144 & 0.886 \\
\hline Pause rate & 0.038 & $(0.010)$ & 0.032 & $(0.096)$ & 3.081 & 0.004 \\
\hline Time per pause & 0.49 & $(0.16)$ & 0.42 & $(0.17)$ & 2.170 & 0.038 \\
\hline Time per key & 0.21 & $(0.019)$ & 0.21 & $(0.044)$ & -0.234 & 0.817 \\
\hline Adjusted time per key & 0.17 & $(0.010)$ & 0.18 & $(0.021)$ & -0.937 & 0.357 \\
\hline Word count & 138.27 & $(9.82)$ & 135.67 & (14.26) & 0.861 & 0.396 \\
\hline Words per sentence & 23.52 & $(4.23)$ & 22.85 & $(3.13)$ & 0.731 & 0.470 \\
\hline Words over 6 letters & 24.29 & $(5.32)$ & 24.75 & $(6.07)$ & -0.327 & 0.746 \\
\hline Correction key rate & 0.092 & $(0.029)$ & 0.091 & $(0.024)$ & 0.097 & 0.924 \\
\hline Function key rate & 0.018 & $(0.0060)$ & 0.021 & $(0.0082)$ & -1.620 & 0.116 \\
\hline Other key rate & 0.89 & $(0.030)$ & 0.89 & $(0.023)$ & 0.431 & 0.670 \\
\hline Lexical diversity & 21.75 & $(2.04)$ & 20.86 & $(2.26)$ & 1.605 & 0.114 \\
\hline Cognitive processes & 15.11 & $(3.67)$ & 16.32 & $(4.99)$ & -1.067 & 0.295 \\
\hline Insight & 2.33 & $(1.88)$ & 2.61 & $(1.98)$ & -0.581 & 0.566 \\
\hline Certainty & 1.05 & $(0.93)$ & 1.17 & $(1.20)$ & -0.409 & 0.685 \\
\hline Tentativeness & 2.44 & $(1.29)$ & 2.62 & $(1.68)$ & -0.475 & 0.639 \\
\hline Perception & 1.14 & $(1.02)$ & 0.74 & $(0.65)$ & 1.750 & 0.091 \\
\hline Positive emotion & 3.64 & $(1.66)$ & 3.52 & $(1.71)$ & 0.314 & 0.756 \\
\hline Negative emotion & 1.13 & (1.19) & 1.63 & $(2.14)$ & -1.075 & 0.291 \\
\hline First-person pronouns & 3.10 & $(3.92)$ & 2.98 & $(4.05)$ & 0.121 & 0.905 \\
\hline Third-person pronouns & 1.52 & $(1.80)$ & 0.73 & $(0.90)$ & 2.3316 & 0.028 \\
\hline
\end{tabular}

Table B-7: Feature statistics for Participant 7 (*p<0.0025). Highlighted variables were selected for the logistic regression model. 


\begin{tabular}{|c|c|c|c|c|c|c|}
\hline \multirow{2}{*}{$\begin{array}{l}\text { Participant } 8 \\
\text { Variables }\end{array}$} & \multicolumn{2}{|c|}{ Control $(\mathrm{N}=30)$} & \multicolumn{2}{|c|}{$\begin{array}{c}\text { Experimental } \\
\quad(\mathrm{N}=30)\end{array}$} & \multicolumn{2}{|c|}{ T-test } \\
\hline & Mean & (SD) & Mean & (SD) & $\mathbf{T}$ & $p$ \\
\hline Total duration & 264.14 & $(29.64)$ & 268.63 & $(34.32)$ & -0.774 & 0.445 \\
\hline Pause rate & 0.066 & $(0.014)$ & 0.065 & $(0.015)$ & 0.400 & 0.692 \\
\hline Time per pause & 0.80 & $(0.20)$ & 0.81 & $(0.27)$ & -0.106 & 0.916 \\
\hline Time per key & 0.25 & $(0.019)$ & 0.25 & $(0.038)$ & -0.349 & 0.729 \\
\hline Adjusted time per key & 0.21 & $(0.0082)$ & 0.21 & $(0.016)$ & -0.406 & 0.688 \\
\hline Word count & 153.07 & $(9.48)$ & 156.57 & $(10.26)$ & -1.525 & 0.138 \\
\hline Words per sentence & 14.68 & $(2.24)$ & 13.84 & $(2.04)$ & 1.582 & 0.125 \\
\hline Words over 6 letters & 13.85 & $(4.23)$ & 12.07 & $(3.64)$ & 1.125 & 0.270 \\
\hline Correction key rate & 0.12 & $(0.022)$ & 0.012 & $(0.022)$ & -0.632 & 0.533 \\
\hline Function key rate & 0.028 & $(0.0047)$ & 0.028 & $(0.0081)$ & 0.004 & 0.997 \\
\hline Other key rate & 0.86 & $(0.023)$ & 0.85 & $(0.023)$ & 0.615 & 0.543 \\
\hline Lexical diversity & 20.90 & $(1.70)$ & 21.16 & $(2.01)$ & -0.532 & 0.597 \\
\hline Cognitive processes & 15.70 & $(3.34)$ & 16.61 & (3.39) & -1.148 & 0.260 \\
\hline Insight & 1.75 & $(1.01)$ & 2.08 & $(1.44)$ & -1.058 & 0.299 \\
\hline Certainty & 1.27 & $(0.92)$ & 1.46 & $(1.12)$ & -0.608 & 0.548 \\
\hline Tentativeness & 2.20 & $(1.42)$ & 2.09 & $(1.72)$ & 0.288 & 0.775 \\
\hline Perception & 1.79 & $(1.36)$ & 1.73 & $(1.27)$ & 0.162 & 0.873 \\
\hline Positive emotion & 2.94 & $(1.92)$ & 3.37 & $(1.92)$ & -0.888 & 0.382 \\
\hline Negative emotion & 1.17 & $(0.94)$ & 1.25 & $(1.04)$ & -0.329 & 0.745 \\
\hline First-person pronouns & 5.24 & $(4.47)$ & 4.86 & $(4.19)$ & 0.340 & 0.735 \\
\hline Third-person pronouns & 3.089 & $(2.88)$ & 3.95 & $(2.66)$ & -1.769 & 0.087 \\
\hline
\end{tabular}

Table B-8: Feature statistics for Participant $8(* p<0.0025)$. Highlighted variable was selected for the logistic regression model. 


\begin{tabular}{|c|c|c|c|c|c|c|}
\hline \multirow{2}{*}{$\begin{array}{l}\text { Participant } 9 \\
\text { Variables }\end{array}$} & \multicolumn{2}{|c|}{ Control $(\mathrm{N}=30)$} & \multicolumn{2}{|c|}{$\begin{array}{c}\text { Experimental } \\
\quad(\mathrm{N}=30)\end{array}$} & \multicolumn{2}{|c|}{ T-test } \\
\hline & Mean & (SD) & Mean & (SD) & $\mathbf{T}$ & $p$ \\
\hline Total duration & 140.55 & $(8.40)$ & 151.16 & $(7.97)$ & -0.727 & 0.473 \\
\hline Pause rate & 0.020 & $(0.0063)$ & 0.018 & $(0.0056)$ & 1.798 & 0.083 \\
\hline Time per pause & 0.20 & (0.068) & 0.17 & $(0.074)$ & 1.504 & 0.143 \\
\hline Time per key & 0.16 & $(0.0075)$ & 0.17 & $(0.060)$ & -0.887 & 0.382 \\
\hline Adjusted time per key & 0.14 & $(0.0062)$ & 0.15 & $(0.022)$ & -0.734 & 0.469 \\
\hline Word count & 160.77 & $(7.96)$ & 158.93 & $(7.92)$ & 0.887 & 0.382 \\
\hline Words per sentence & 20.13 & $(3.78)$ & 20.04 & $(3.57)$ & 0.096 & 0.924 \\
\hline Words over 6 letters & 11.58 & (3.39) & 12.04 & $(4.84)$ & -0.422 & 0.676 \\
\hline Correction key rate & 0.044 & $(0.020)$ & 0.040 & (0.023) & 0.730 & 0.471 \\
\hline Function key rate & 0.022 & $(0.0045)$ & 0.019 & $(0.0048)$ & 2.553 & 0.016 \\
\hline Other key rate & 0.93 & $(0.021)$ & 0.94 & $(0.023)$ & -1.248 & 0.222 \\
\hline Lexical diversity & 22.62 & $(2.49)$ & 23.31 & $(1.64)$ & -1.274 & 0.208 \\
\hline Cognitive processes & 20.46 & (3.68) & 19.97 & (3.69) & 0.525 & 0.604 \\
\hline Insight & 2.58 & $(1.51)$ & 2.52 & $(1.47)$ & 0.172 & 0.865 \\
\hline Certainty & 1.70 & $(1.30)$ & 1.88 & $(1.36)$ & -0.526 & 0.603 \\
\hline Tentativeness & 3.84 & $(1.82)$ & 3.67 & $(2.04)$ & 0.418 & 0.679 \\
\hline Perception & 1.13 & $(0.97)$ & 1.19 & $(1.20)$ & -0.227 & 0.822 \\
\hline Positive emotion & 3.63 & $(2.07)$ & 3.38 & $(1.76)$ & 0.468 & 0.643 \\
\hline Negative emotion & 1.92 & $(0.95)$ & 2.29 & $(1.86)$ & -1.008 & 0.322 \\
\hline First-person pronouns & 3.99 & $(4.49)$ & 3.94 & $(4.20)$ & 0.045 & 0.965 \\
\hline Third-person pronouns & 1.10 & $(1.72)$ & 0.97 & $(1.06)$ & 0.362 & 0.720 \\
\hline
\end{tabular}

Table B-9: Feature statistics for Participant $9(* p<0.0025)$. Highlighted variable was selected for the logistic regression model. 